MLM-2450

UC -4 and UC-22

\title{
Mound Laboratory Activities in Chemical and Physical Research: January-June 1977
}

Issued: October 4, 1977

PPINTED IN THE UNITED STATES OF AMERICA

Available from

National Technical Information Sarvice

U. S. Depertment of Commerce

6285 Port Royal Road

Springfleld, Virginis 22161

Price: Printed Copy 100 ; Microfiche $\$ 3.00$

5.25

\section{MONSANTO RESEARCH CORPORATION}

A Subsidiary of Monsanto Company

\author{
MOUND LABORATORY \\ Miamisburg, Ohio \\ 45342 \\ operated for

\section{UNITED STATES ENERGY RESEARCH AND DEVELOPMENT ADMINISTRATION}

U. S. Government Contract No. EY-76-C-04-0053

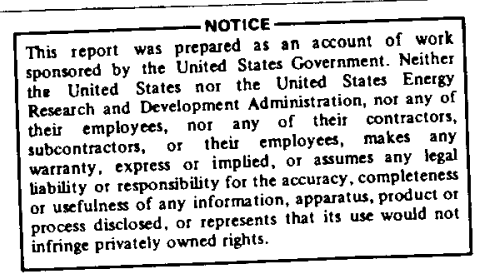




\section{DISCLAIMER}

This report was prepared as an account of work sponsored by an agency of the United States Government. Neither the United States Government nor any agency Thereof, nor any of their employees, makes any warranty, express or implied, or assumes any legal liability or responsibility for the accuracy, completeness, or usefulness of any information, apparatus, product, or process disclosed, or represents that its use would not infringe privately owned rights. Reference herein to any specific commercial product, process, or service by trade name, trademark, manufacturer, or otherwise does not necessarily constitute or imply its endorsement, recommendation, or favoring by the United States Government or any agency thereof. The views and opinions of authors expressed herein do not necessarily state or reflect those of the United States Government or any agency thereof. 


\section{DISCLAIMER}

Portions of this document may be illegible in electronic image products. Images are produced from the best available original document. 


\section{Foreword}

This report is issued semiannually by Mound Laboratory. Under the sponsorship of the ERDA Divison of Basic Energy Science, Mound Laboratory is responsible for producing materials for use in the physical sciences to further the progress of science and technology in the public interest. Additional research activities of related interest under the sponsorship of the Division of Military Application are also reported here.

This report is submitted by W. T. Cave, Director of Nuclear Operations, and R. E. Vallee, Manager of Technology Applications and Development, from contributions prepared by W. M. Rutherford, Science Fellow (Thermal Diffusion); W. L. Taylor, Science Fellow (Gas Dynamics and Cryogenics); G. L. Silver, Senior Research Specialist (Separation Chemistry); R. C. Bowman, Leader, Metal Hydride Research, and from members of the Isotope Separation Section: R. A. Schwind, Isotope Separation Manager; E. D. Michaels, Leader, Isotope Separations Development; W. J. Roos, Leader, Stable Isotopes Production; B. E. Jepson, Leader, Metal Isotope Separation; R. M. Watrous, Leader Radioisotopes Separation; and V. L. Avona, Leader, Stable Isotope Sales.

These reports are not intended to constitute publication in any sense of the word. Final results will either be submitted for publication in regular professional journals or be published in the form of MLM topical reports.

P. A. Semple, Editor 


\section{Contents}

\section{Computer Operations}

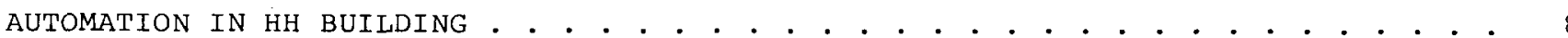

Several automation projects completed in $\mathrm{HH}$ Building include enhancements to the MODCOMP IV computer system, the installation of an automatic inlet system on the CEC 620 mass spectrometer, startup of the neon monitoring system, computer-assisted control of a quadrupole mass spectrometer, and computer-assisted control of the beam scattering chamber.

\section{Isotope Separation}

ARGON

Production of $7 \%$ argon-38 and $99.5 \%$ argon-36 continued during this period.

CARBON. . . . . . . . . . . . . . . . . . . . . . . . . . . . . . . . . . . . .

A 45-gram batch of $99 \%$ carbon-13 was produced by conversion from carbon dioxide to elemental carbon.

HELIUM-3. . . . . . . . . . . . . . . . . . . . . . . . . . . . . . . .

Approximately 3000 STP liters of $99.9 \%$ helium-3 were produced.

The nine-column and 19-column cascades continued to produce $99 \%$ krypton-86.

The 10-column cascade produced a second batch of $90 \%$ krypton-80.

Batches of neon-20 and neon-22 which are slightly below product specifications were upgraded. 
The oxygen electrolysis system is being modified to facilitate the production of oxygen-17 and -18 gas.

The following approximate quantities of materials were produced: 0.65 STP liter of $40 \%$ xenon-124; 6.0 STP liters of $2 \%$ xenon-126; and 10.0 STP 1 iters of $80 \%$ xenon-136.

\section{Low Temperature Research}

HELIUM VIRIAL EQUATION. . . . . . . . . . . . . . . . . . . . . . . . . . . 14

Data for the second virial coefficient, B, of helium are compared with calculations of $B$ from seven potential functions in the temperature region from 1.5 to $600 \mathrm{~K}$. Recent measurements have narrowed the uncertainty in B considerably. While most recent potentials do not satisfactorily fit the data over the entire temperature range, the one exception is an unpublished potential measured by Aziz and co-workers. This potential was used to calculate $\mathrm{B}$ for ${ }^{3} \mathrm{He}$ over the whole temperature range since data exist only between 1.5 and $4.2 \mathrm{~K}$.

\section{Metal Hydride Research}

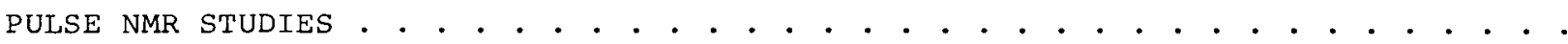

Studies of $\mathrm{TiFeH}_{\mathrm{N}}$ and Related systems Proton spin echo measurements were performed on $\beta$ and $\gamma$ phase samples of $\mathrm{TiFeH}_{\mathrm{x}}$. Motional narrowing (i.e., $\mathrm{T}_{2}{ }^{\prime}$ increases) were observed above $360 \mathrm{~K}$ and $390 \mathrm{~K}$ for the $\beta$ and $\gamma$ phases, respectively. Both phases yielded an activation energy of $0.26 \pm 0.0 \mathrm{leV}$ for hydrogen diffusion.

Diffusion Studies in $V_{x}$ Pulse NMR techniques are being used to evaluate the phase transformation and diffusion processes in $V_{x}$. Proton $T_{1}$ relaxation times have been measured between $100 \mathrm{~K}$ and $500 \mathrm{~K}$ for several $\mathrm{VH}_{\mathrm{x}}$ compositions. Although analyses of $\mathrm{T}_{1}$ are complicated by phase transitions, the diffusion characteristics in the b.c.t. B-phase have been determined. The composition dependencies of $E_{a}$ and $D_{0}$ cannot be explained by the vacancy model proposed for f.c.c. hydrides. An alternative explanation based upon localized disorder is presented. 
ELECTRICAL RESISTIVITY STUDIES OF PHASE TRANSFORMATIONS IN VH . . . . . . . . . 26

Phase properties of $\mathrm{VH}_{\mathrm{x}}$ alloys, with $\mathrm{x}=0.40-0.85$, are being studied using electrical resistivity. previously unreported transformations of $\mathrm{VH}_{0} .80$ were detected at 178,344 , and $433 \mathrm{~K}$. The $\mathrm{VH}_{0} .80$ transformation at $433 \mathrm{~K}$ differed from those observed near the same temperature for lower compositions. Vanadium deuteride and tritide are also to be studied.

ELECTRON PARAMAGNETIC RESONANCE STUDIES OF TRANSITION METAL IONS IN LITHIUM HYDRIDE . • • . . . . . . . . . . . . . . . . . . . . . . . . . . . . 29

Rhodium Ions in LiH and LiD Single crystals Analysis of the electron paramagnetic resonance (EPR) spectra for $\mathrm{Rh}^{+2}$, species $A$, in LiH and LiD single crystals has been completed. It shows that $A$ is a nearly

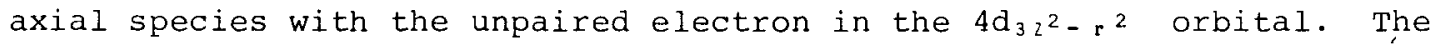
total magnetic spin density on the axial ligands is $9 \%$

Nickel Ions in LiH Single Crystals preliminary interpretation of the EPR spectra of LiH containing nickel ions indicates that nickel is present as two species--one axial and one isotropic. The oxidation state for both is probably $\mathrm{Ni}^{+}{ }_{1}$.

BAND THEORY AND ELECTRONIC STRUCTURE. . . . . . . . . . . . . . . . . . . . . . . 30

Computer codes required for a molecular quantum mechanical study of interstitial metal hydrides are now being implemented using Brookhaven's CDC 6600 and 7600 computers. Six major programs, as well as various minor utility programs, have been implemented and tested; work is currently proceeding on the restricted Hartree-Fock (RHF)/restricted Generalized Valence Bond (GVB) program. Calculations on prototype metal and metal hydride clusters will ensue. 


\section{Separation Chemistry}

PROBLEMS IN PLUTONIUM CHEMISTRY (V): THE SOLUBILITY OF TETRAVALENT PLUTONIUM HYDROXIDE. . . . . . . . . . . . . . . . . . . . . . . . . . . . . 33

The unsuspected presence of pentavalent plutonium may contribute to errors in determining the solubility of tetravalent plutonium hydrous oxide in both laboratory and environmental circumstances. Empirically determined alpha coefficients are presented as one means of approximating the oxidation state distributions.

PROBLEMS IN PLUTONIUM CHEMISTRY (VI): KINETICS OF PLUTONIUM DISPROPORTIONATION REACTIONS. . . . . . . . . . . . . . . . . . . . . . . . . . . . 34

The possiblity of characterizing the kinetics of plutonium disproportionation reactions by determining the instantaneous concentrations of two plutonium oxidation states is illustrated.

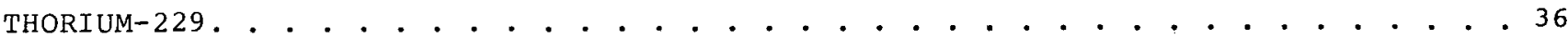

A total of $11.2 \mathrm{mg}$ of thorium-229 was separated from low-purity aged uranium-233. A new, redesigned extraction apparatus was installed and used. A different technique for dissolving high-purity $\mathrm{UF}_{4}$ proved to be very effective.

THORIUM-230 AND PROTACTINIUM-231 . . . . . . . . . . . . . . . . . . . . . . . . 36

A shipment of $31.0 \mathrm{~g}$ of thorium-230 was made to Isotope Sales at oRNL in partial fulfillment of the FY-1977 quota. An additional $31.26 \mathrm{~g}$ of thorium230 is on hand toward completion of the FY-1977 quota. Approximately $210 \mathrm{mg}$ of protactinium-231 is being purified.

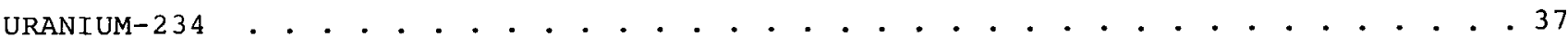

The initial separation, by oxalate precipitation, of an estimated $5.4 \mathrm{~g}$ of uranium-234 from $124.3 \mathrm{~g}$ of aged plutonium-238 was completed. A total of $4.4 \mathrm{~g}$ of uranium-234 underwent the intermediate separation by anion exchange in nitrate media. 


\section{Separation Research}

LIQUID THERMAL DIFFUSION. . . . . . . . . . . . . . . . . . . . . . . . . . 40

A 61-cm experimental column was used to measure isotopic speparations of methyl chloride and sulfur dioxide. Reduced thermal diffusion factors for these two compounds, which have critical temperatures close to the hot wall temperature of the column, were found to be 1.46 and 1.42 , respectively. The physical properties necessary to reduce the separation data were estimated by several empirical techniques. The quantity DVn/RT, where $D$ is the diffusion coefficient, $V$ the molar volume, $n$ the viscosity, $R$ the gas constant, and $\mathrm{T}$ the temperature, was postulated to be a function only of the reduced density in the critical region.

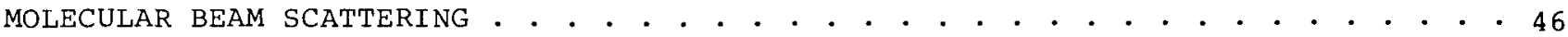

Ideal skimmers and precision nozzles were installed on both sources in the molecular beam system for greater beam intensity. Final laser alignment of the primary and secondary chamber arms with the detector platform was completed.

TRANSPORT PROPERTIES. . . . . . . . . . . . . . . . . . . . . . . . . . . . . 4

Calibration of the thermometers in the two-bulb apparatus, employed in the determination of the thermal diffusion factor $\left(\alpha_{\mathrm{T}}\right)$ for ${ }^{3} \mathrm{He}-{ }^{4} \mathrm{He}$, revealed a possible inaccuracy in the cold-bulb temperature for some of the earlier data points. Three current experiments failed to reproduce the earlier $\alpha_{T}$ values; the method of estimating the relaxation time for an experiment may be a partial explanation. 


\section{Computer Operations}

\section{Automation in $\mathrm{HH}$ Building}

ENHANCEMENTS TO THE MODCOMP COMPUTER SYSTEM

During the past year, $128 \mathrm{~K}$ bytes of $16-\mathrm{bit}$ core memory have been added to the central system. This brings to $256 \mathrm{~K}$ bytes the total available in the multiprogramming environment. Of this total, approximately $50 \mathrm{~K}$ bytes are used by the MAX IV executive software. The remainder has been adequate for the simultaneous operation of a batch stream, mass spectrometer task, neon monitoring task, and either the inventory data base task or the scattering chamber task.

The next release of MAX IV is scheduled to include a new FORTRAN run-time package which will allow recursive entries. This will reduce the size of all FORTRAN programs by about $16 \mathrm{~K}$ bytes and thus allow even more programs to be executed concurrently.

Also, a peripheral controller interface (PCI) has been added to allow further $I / O$ expansion. So far, the PCI has been interfaced to two full-duplex, serial channels for communication with a microcomputer network which is in the design stage.

AUTOMATIC INLET FOR THE CEC 620 MASS SPECTROMETER

To achieve further computer control of the model 620 mass spectrometer, which is used for process analyses, an automatic inlet system was designed and built. This system, controlled either manually or by computer, admits gas samples from batch, standard, and remote ports. As shown in Figure 1, the system consists of two gas volumes which are interconnected via a solenoid valve. The batch and standard containers are connected to one 2-1iter tank through double solenoid valve ports, and the remote sampling capillaries are attached to the second 2-liter tank through single solenoid valve ports. This double tank arrangement allows gas to be sampled, via capillary tubing from a production separation system, into a single 2-liter volume. This is desirable in systems permitting only a very small volume of sampled gas. On batch and standard analyses, the full 4-liter volume is used. All of the sample inlet valves are Skinner \#V52 solenoid valves. Each tank has its own connection to a mechanical roughing pump and a connection to an oil diffusion pump. These four valves and the valve to the ionizer are VACOA Sov high vacuum solenoid valves $(0.718 \mathrm{in}$. diameter orifice).

Each of the solenoid valves is activated through a solid state relay which allows for a minimum on-off cycle time of 20 msec. The double valve arrangement on the batch and standard ports was necessary to control the rate of flow of gas when the gas was admitted under computer control. A single valve cannot be operated fast enough to reliably acquire the quantity of gas needed (i.e., normally a pressure of 100 microns in the 4-liter volume) for an analysis. Controlling the flow rate on the remote ports is effected by proper choice of capillary size. 


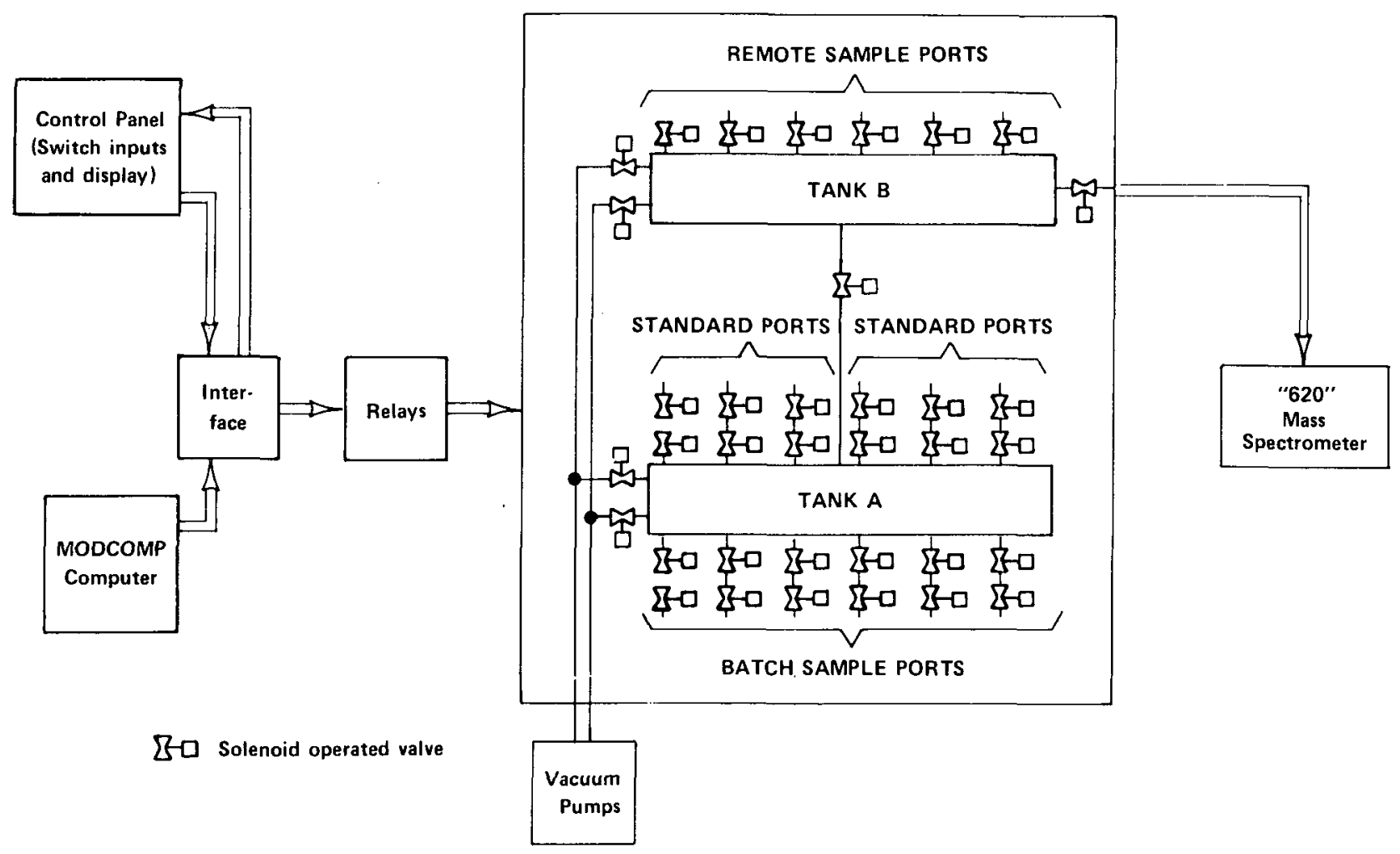

FIGURE 1 - Automatic inlet system for cycloid mass spectrometer.

The inlet system can be controlled remotely using subroutines that have been added to the mass spectrometer program in the MODCOMP IV computer system. The program has full control of the inlet system and can sample any of the ports to bring the inlet tanks to the desired operating pressure. Each valve is controllable singly or in any combination via simple commands entered through the control terminal.

MONITORING OF THE NEON PRODUCTION SYSTEM

The neon separation system is now being monitored under computer control. The new system monitors the neon composition, measures temperatures, and measures pressures at critical locations in the system. Neon is sampled, via solenoid valves, at the top, bottom, and side product loops and from the feed line.
The valves are connected to capillary tubing which transports the gas to the mass spectrometer, via the automatic inlet system described above in the section on the 620 mass spectrometer. The gas sampling and mass analyses are perforied under control of the mass spectrometer software. The results of the gas analyses are used in the formulation of the draw-off rates for each of the three product points.

The temperature and pressure monitoring electronics are shown in Figure 2. The water jacket temperature of each of the four columns, as well as the ambient room temperature, is measured with thermistor probes. As shown in Figure 2, the probe signals are routed through an

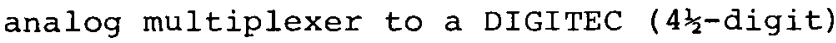
panel meter for local indication of the 


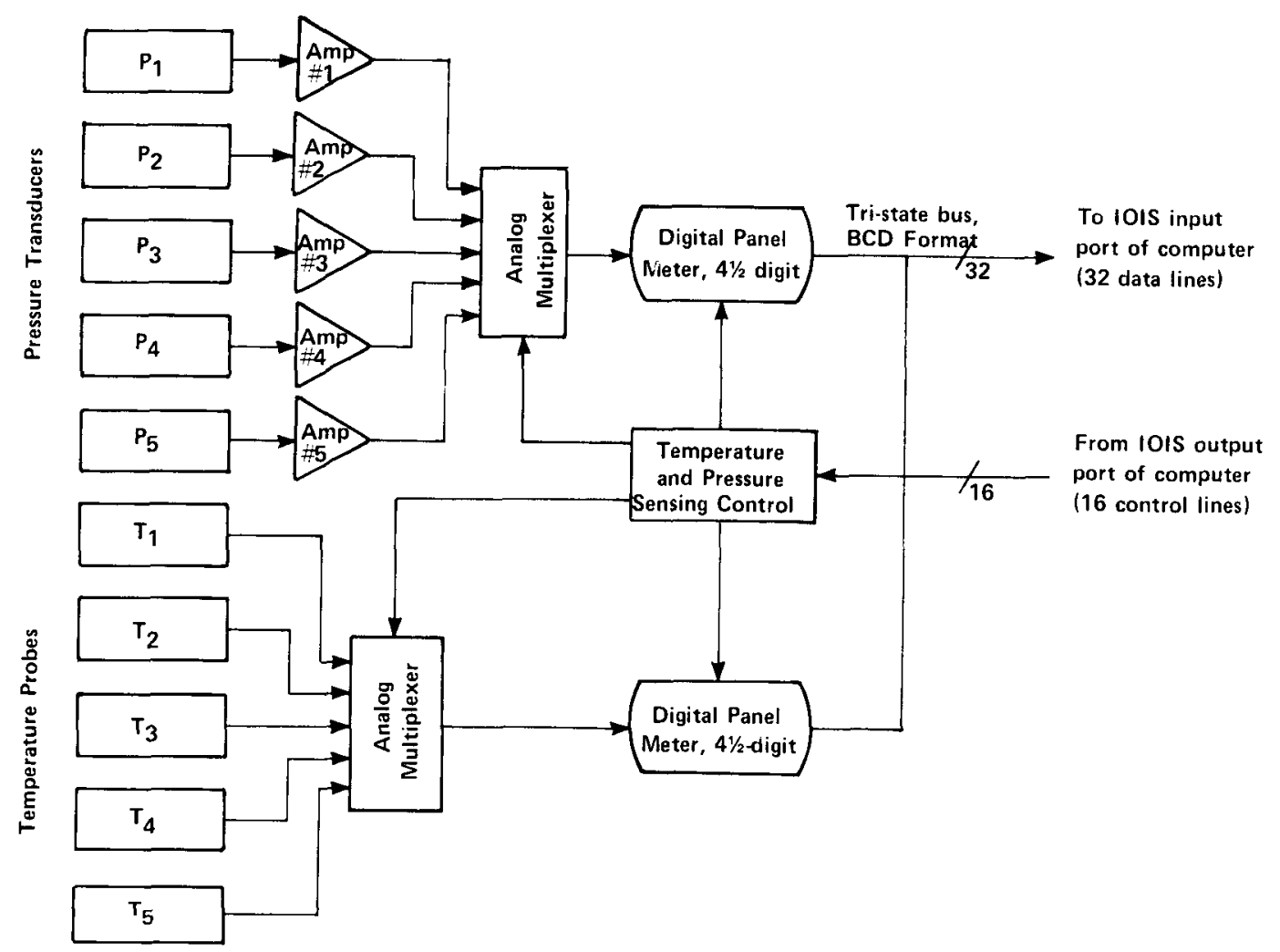

FIGURE 2 - Temperature and pressure monitoring electronics for the neon system.

temperature. The digitized values are available on a parallel BCD (binary coded decimal) bus for routing to the computer system. The temperatures can be read under manual or computer control.

Pressure transducers have been installed at five points in the system. These allow the pressure to be measured in the feed tank, three product tanks, and the separation system. The pressure transducers are Sensotec, Model TJE, with a $\pm 0.1 \%$ accuracy. They are connected to Sensotec, Series 7, strain gauge amplifiers. Each amplifier output is routed, through an analog multiplexer, to a digital panel meter in the same manner as the temperature signals. The digitized information is available on the parallel $B C D$ bus for use by the computer.
There are two computer programs used for monitoring of the neon system. The program for operating the mass spectrometer (MS) has been expanded to include subroutines that acquire neon gas samples via capillary tubing connected to the MS automatic inlet system. Currently, $1 \mathrm{~cm}^{3}$ of gas at STP is removed from the production system for each analysis. This volume is smaller than the volume removed during manual sampling by a factor of 2 to 5. The volume can be reduced further, by a factor of 2 to 3 , with suitable changes in the system. After each neon mass analysis, the capillary system is flushed with helium and then pumped out before the next neon sample is introduced. This technique removes $>99 \%$ of the previous sample. 
A second monitoring program runs continuously in the computer system. Its function is to sample periodically all five temperature readings and five pressure transducers at intervals chosen by the operator. These values are stored, along with the times they were taken, on a disk file which can hold the most recent 150 sets of data. The operator can access any of this information in tabular or graphical form as a function of time.

For each set of data, the system mass balance is calculated based on the temperature and pressure parameters and on the known volumes of each part of the system. The mass balance number is useful for determining flow rates into each of the product tanks. Also, by comparing these rates with the feed tank rate, leaks into or out of the system can be spotted.

COMPUTER CONTROL OF A QUADRUPOLE MASS SPECTROMETER

The current revision (MS5) of the computer software for mass spectrometer control has been expanded and upgraded to include control of the Extranuclear quadrupole mass spectrometer as well as the CEC 620 cycloidal mass spectrometer. Hardware interfacing includes a 16-bit parallel interface which provides sweep circuit information, connection of the detector to the computer analog input subsystem, $X-Y$ analog signals for an oscilloscope display, and $X-Y$ analog signals for driving an $X-Y$ recorder.

Computer control of the sweep circuit is accomplished by connecting the 16-bit output from a computer port to a 16-bit, temperature-controlled digital-to-analog converter (DAC). This DAC provides a 0 to $10 \mathrm{~V}$ dc analog signal which is coupled to the control circuit of a KEPCO power supply. This power supply provides the proper dc bias for the quadrupole. The circuit is designed to allow incremental mass steps of $2.3 \mathrm{millimass}$ units.

The electron multiplier detector of the spectrometer is connected to the analog input system of the computer. This is an autoranging system that provides a fullscale measurement capability from $\pm 5 \mathrm{mV}$ to $\pm 10.24 \mathrm{~V}$ with up to 15 -bit resolution.

The program will control whichever spectrometer the operator specifies. Separate data sets are maintained on the disk for information such as sensitivity factors, natural abundance values, and the parameters for mass calibration. The computer is now capable of controlling variables such as the sweep rate and mass resolution, sweeping multiple mass regions on a single run, acquiring the raw data and analyzing the data for net peak heights. This information is then used by the computer for determining relative mole fractions of the particular species, and presenting these data in report form. An early version of this program has been described previously [1].

\section{AUTOMATION OF BEAM SCATTERING CHAMBER}

Computer hardware and software have been developed to assist in operation of the beam scattering chamber. A Remote Acquisition subsystem (REMAC) provides the hardware interface to the MODCOMP computer system. REMAC-to-computer transfers are accomplished using a fullduplex serial link with two coaxial cables than can be up to 10,000 feet in length.

A beam detector, consisting of a quadrupole mass filter mounted on a turntable, has 
been developed by the Atomic Beams Group for the beam chamber. This assembly is driven by a stepping motor and allows the detector to be positioned at both positive and negative angles with respect to the primary beam while maintaining a constant radius from the target cell. Computer interfacing has been completed for computer control of turntable positioning, angle readout in digitized form from a synchro converter, and data acquisition from the mass filter detector system. The control software is operational for these functions and contains subroutines that can be called by FORTRAN for access to all REMAC ports.

A project is currently under way for implementing computer control of mass selection by the mass filter. This requires an interface to the REMAC system consisting of a 16-bit, temperaturecontrolled DAC which is used, in turn, to produce a 0 to $10 \mathrm{~V}$ dc signal for the control circuit of the power supply which provides the correct quadrupole dc pole bias. The design and construction of this hardware have been completed and will be tested in the near future.

A diagnostic program has been written in FORTRAN for exercising all REMAC digital and analog I/O channels. This is useful for checking both the REMAC hardware and the interfacing hardware for proper operation. (Richard E. Eppley) 


\section{Isotope Separation Argon}

The 13-column hot wire thermal diffusion cascade has continued to produce approximately $7 \%$ argon-38. This material is being collected and saved for later enrichment to $95 \%$. The system also produced argon-36 at $99.5 \%$ enrichment as a by-product. (Gilbert E. Stuber, Jr.)

\section{Carbon}

A 45-gram batch of $99 \%$ carbon-13 was produced by conversion from carbon dioxide to elemental carbon. The conversion resulted in no detectable degradation in the isotopic composition, the composition being $99.7 \%$ carbon-13 in both the feed and product. The conversion system has been slightly modified to improve the ease and safety of operation. (Gilbert E. Stuber, Jr.)

\section{Helium-3}

During this period approximately 3000 STP liters of $99.9 \%$ helium-3 were produced. (William J. Roos)

\section{Krypton}

The nine-column and 19-column thermal diffusion cascades continued to enrich krypton-86 to $99 \%$. The 10-column cascade produced a second batch of krypton-80 enriched to greater than $90 \%$.

(Gilbert E. Stuber, Jr.)

\section{Neon}

Upgrading of neon-20 and neon-22 to product grade continued in a four-column thermal diffusion system. The material being processed is slightly below product specifications. (Gilbert E. Stuber, Jr.)

\section{Oxygen}

The oxygen electrolysis system is being modified to decrease its holdup volume. This will facilitate the electrolysis of water enriched in either oxygen-17 or oxygen-18 for which much smaller quantities of feed material are available than for oxygen-16. (Gilbert E. Stuber, Jr.)

\section{Xenon}

During this period a 19-column thermal diffusion system produced approximately 0.65 STP liter of $40 \%$ xenon-124; a 24column system produced approximately 6.0 STP liters of $2 \%$ xenon-126; and an eightcolumn system produced approximately 10.0 STP liters of $80 \%$ xenon-136.

(William J. Roos) 


\section{Low Temperature Research Helium Virial Equation}

The representation of the second virial coefficient, B, for helium has presented an interesting problem for some time. Going back to the original Berlin and Leiden work, the data for $B$ between 80 and $600 \mathrm{~K}$ show a considerable spread at any given temperature. The magnitude appears to have increased with time as shown at $0^{\circ} \mathrm{C}$ in Table 1 . Because of this state of affairs, comparison of calculations based on realistic potential functions with the measured second virial was not considered very fruitful. In trying to determine a good representation for the second virial of helium, it was noted in Keller's book on helium that the only second virial data for ${ }^{3} \mathrm{He}$ were at very low ( 1.5 to $4.2 \mathrm{~K}$ ) temperatures [2]. It was also seen that an attempt to fit both the ${ }^{3} \mathrm{He}$ and ${ }^{4} \mathrm{He}$ data into that temperature range with a single potential function failed. At that time the Lennard-Jones potential function fit neither set of data, and the Mason-Rice fit ${ }^{4} \mathrm{He}$, but not ${ }^{3} \mathrm{He}$. In the National Bureau of Standards (NBS) compilation of second virials, helium was the one gas for which a recommended potential was not given.

Many potentials have been proposed to fit the properties of helium since it was found tinat a form of the Lennard-Jones was inadequate. In general, these potentials have not been used to calculate second virials because they do not compare with experimental data to sufficient precision to make a quantitative test over an extended temperature range. The situation as of 1972 is shown in Figure 3. The two sets of early data are those of Holborn and otto [3] and Michels and
Wouters [4]. All the other data are post1950 data. The curves in the figure are calculated from two programs: 1) a semiclassical WKB* calculation in the high temperature region $(T>180 \mathrm{~K})$, and 2 ) a full quantum mechanical calculation in the low temperature region ( $1.5<\mathrm{T}<180 \mathrm{~K}$ ). The Lennard-Jones curve was calculated using the deboer parameters [5] for helium. It clearly does not represent the modern data. Potentials constructed to fit other properties of helium are given by Beck [6] and Bruch-McGee [7] (MVDD-1 and MVDD-2, respectively). The MVDD-2 potential was constructed when it was found MVDD- 1 had a well depth, $\varepsilon$, which was too large. The curves for the Beck potential and MVDD-2 appear to fall below the data above $170 \mathrm{~K}$. The figure also shows the NBS compilation [8] curve which has a crossover from high to low compared to the data in the region between 200 and $400 \mathrm{~K}$.

Recently, Lee and co-workers [9, 10 ] have produced potentials for helium from molecular beam scattering. The first potential (labeled Beam 1) is three segments: Exponential, Morse, and van der wals joined by spline functions (ESMSV II). The potential was determined using the low temperature virial for "He. Thus, there is a good fit to the low temperature ${ }^{4} \mathrm{He}$ data shown in Figure 4. It is seen that the Lee (Beam l) potential also fits the ${ }^{3}$ He data quite well. Looking at Figures 3 and 4 , the Lennard-Jones and Beck curves are high at low temperatures and low at high temperatures. If anything, the Lee (Beam 1) curve is high at high temperatures (Figure 3). So far, then, none of the potentials has really satisfactorily fit the data.

ॠWentzel-Kramers-Brillouin 
Table 1 - VALUES OF THE SECOND VIRIAL-

COEFFICIENT (B) FOR HELIUM AT $\mathrm{T}=0^{\circ} \mathrm{C}$

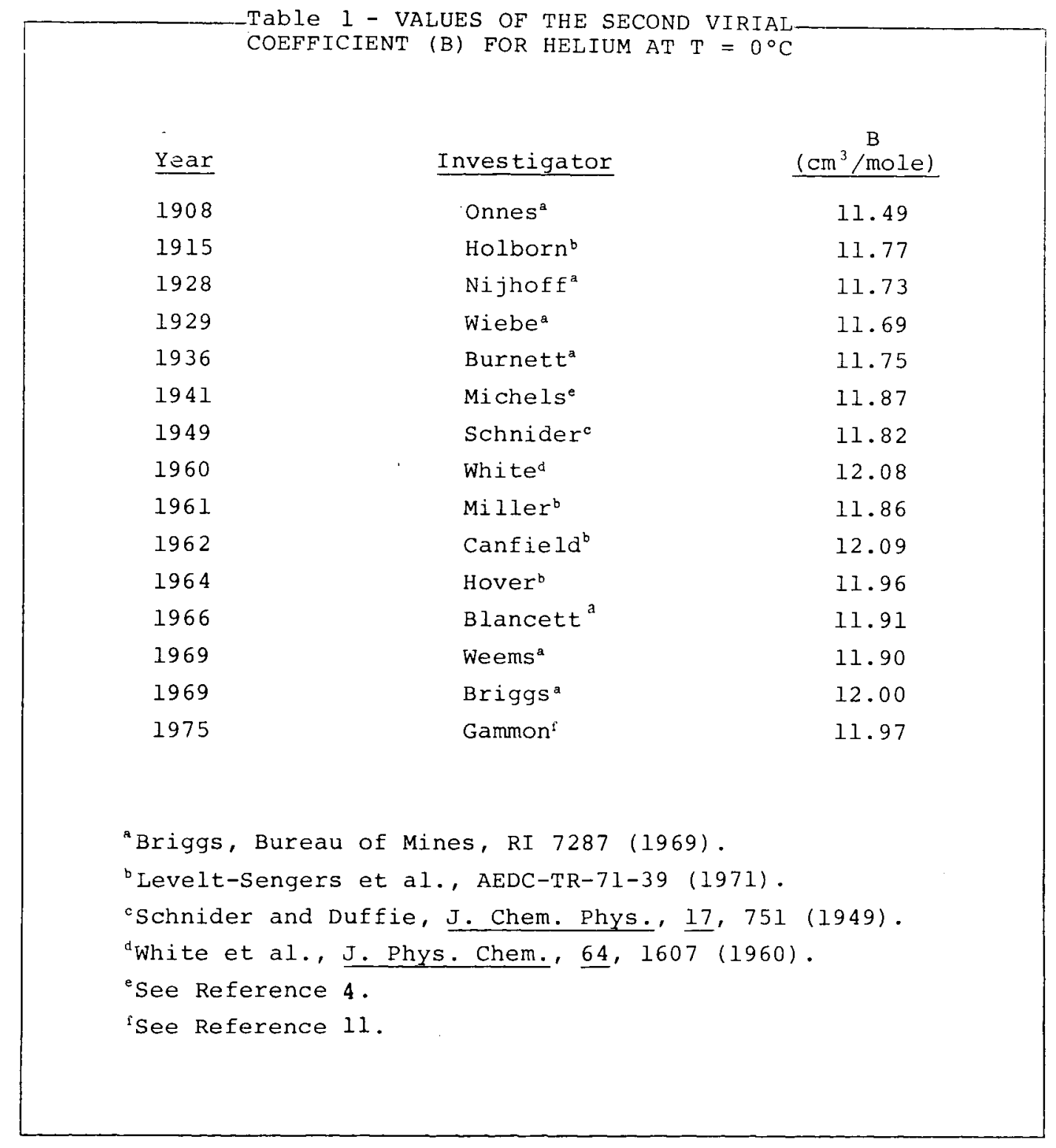




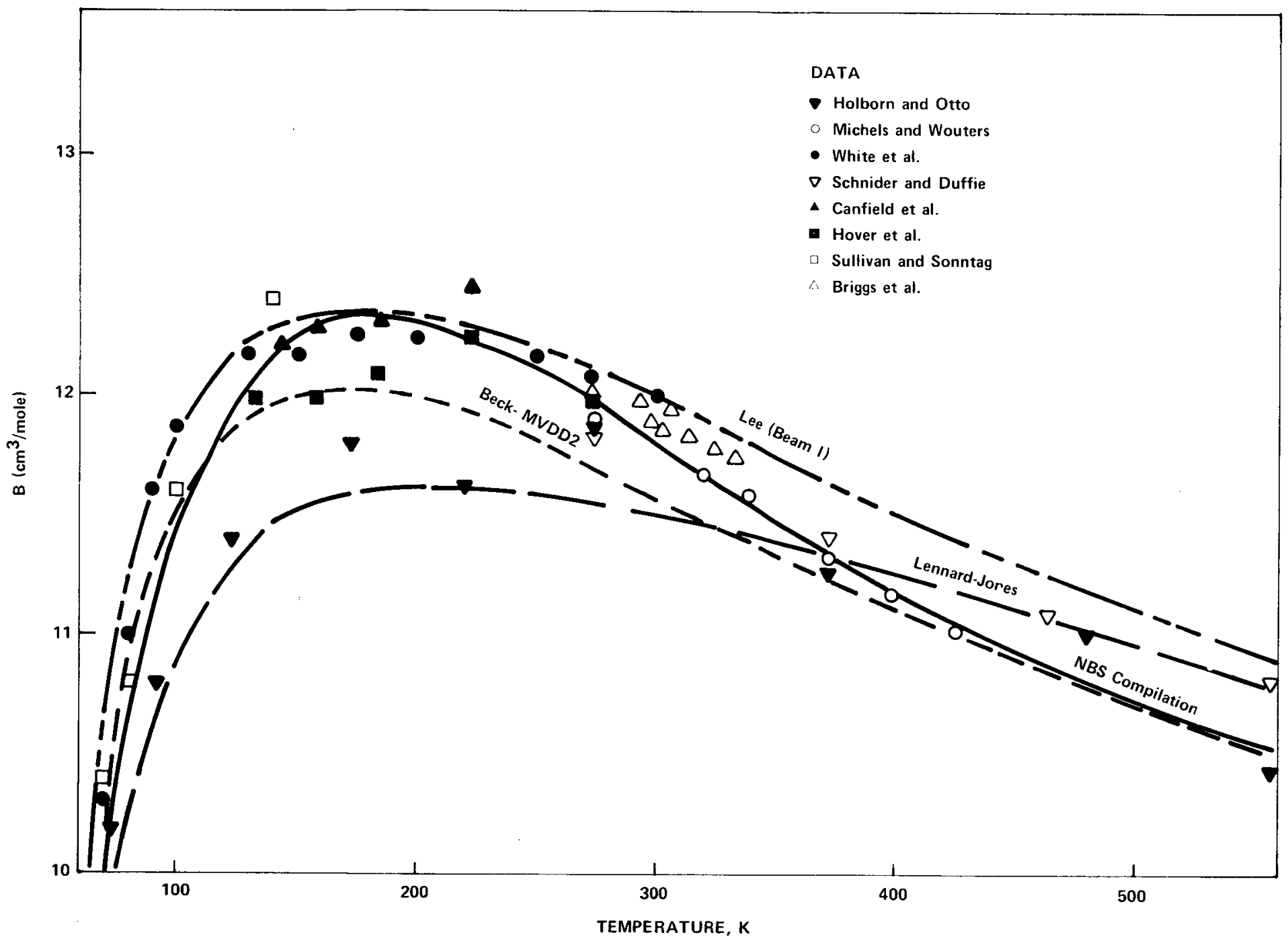

FIGURE 3 - Comparison of second virial data for ${ }^{4}$ He with second virial calculated from several potential functions. 


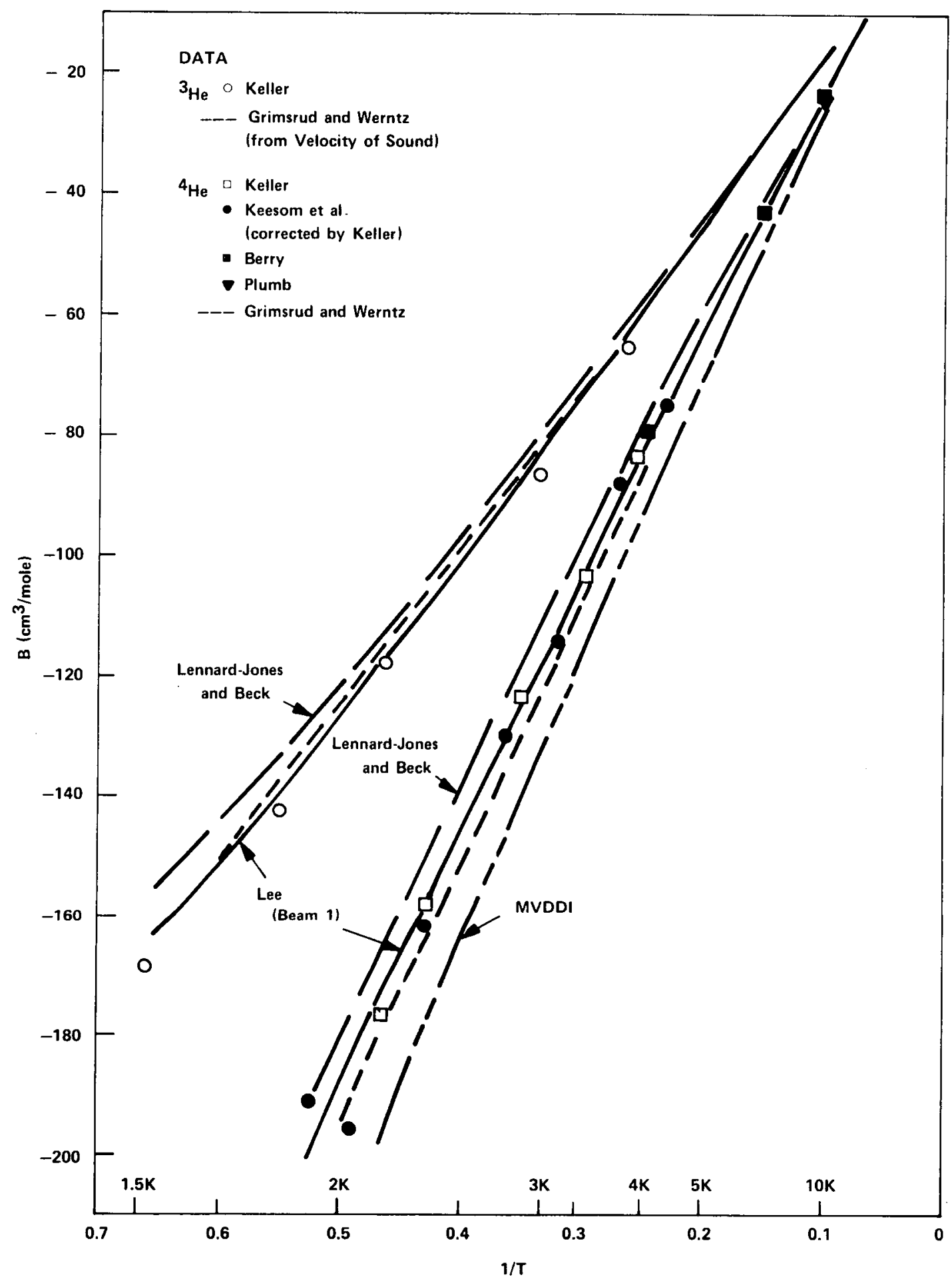

FIGURE 4 - Comparison of low-temperature second virial data for ${ }^{3}$ He and ${ }^{4} \mathrm{He}$ with second virial calculated from several potential functions. 
Ignoring the data of Holborn and otto and that of Michels and Wouters and adding the second virial calculated from Gammon's [11; velocity of sound data results in the data in Figure 5. The data now exhibit a considerably smaller spread in values. The Lee ESMSV II (Beam 1) curve is reproduced from Figure 4. Also shown is the curve for the MVDDl potential of Bruch and McGee which produces a very good representation of the high temperature data, but not of the low temperature data. In a later paper, Lee et al. [10] give a revised potential ESMMSV (Beam 2) for helium. Figure 5 shows that this potential produces a good fit above $250 \mathrm{~K}$ but gets progressively worse at lower temperatures.

These piecewise connected potentials have turned out to be difficult to work with. Thus, in the last two years, workers have tried to construct potentials using only the well-characterized exponential repulsive portion and a complete description of the van der waals attractive portion. There is an electron overlap region in between these two portions, however, which has been hard to describe. Toennies [12] and Ahlrich [13] have worked on these forms. Ahlrich produces a curve the same as ESMSV II (Beam I). Aziz [14], in work to be published, has slightly modified Ahlrich's potential. As seen in Figures 5 and 6 , Aziz's potential gives the best representation of the second virial for ${ }^{4} \mathrm{He}$ from 1.5 to $600 \mathrm{~K}$. The deviation between this potential and Gammon's data is less than $0.02 \mathrm{~cm}^{3} /$ mole between 100 and $400 \mathrm{~K}$.

Thus, the second virial for ${ }^{3} \mathrm{He}$ at high temperatures can now be calculated with some confidence. The calculated values for ${ }^{3}$ He using Aziz's potential are shown in Figure 5. The quantum difference between ${ }^{3} \mathrm{He}$ and ${ }^{4} \mathrm{He}$ amounts to $0.2 \mathrm{~cm}^{3} /$ mole at $300 \mathrm{~K}$. Knowing $B$ to the above accuracy for ${ }^{4} \mathrm{He}$ also allows a more accurate determination of the nigher virials for helium. In particular, an accurate calculation of the third virial using Aziz's potential can be compared with the measured third virials to determine the fraction of three-body collisions contributing to the third virial. (George T. McConville and William L. Taylor) 


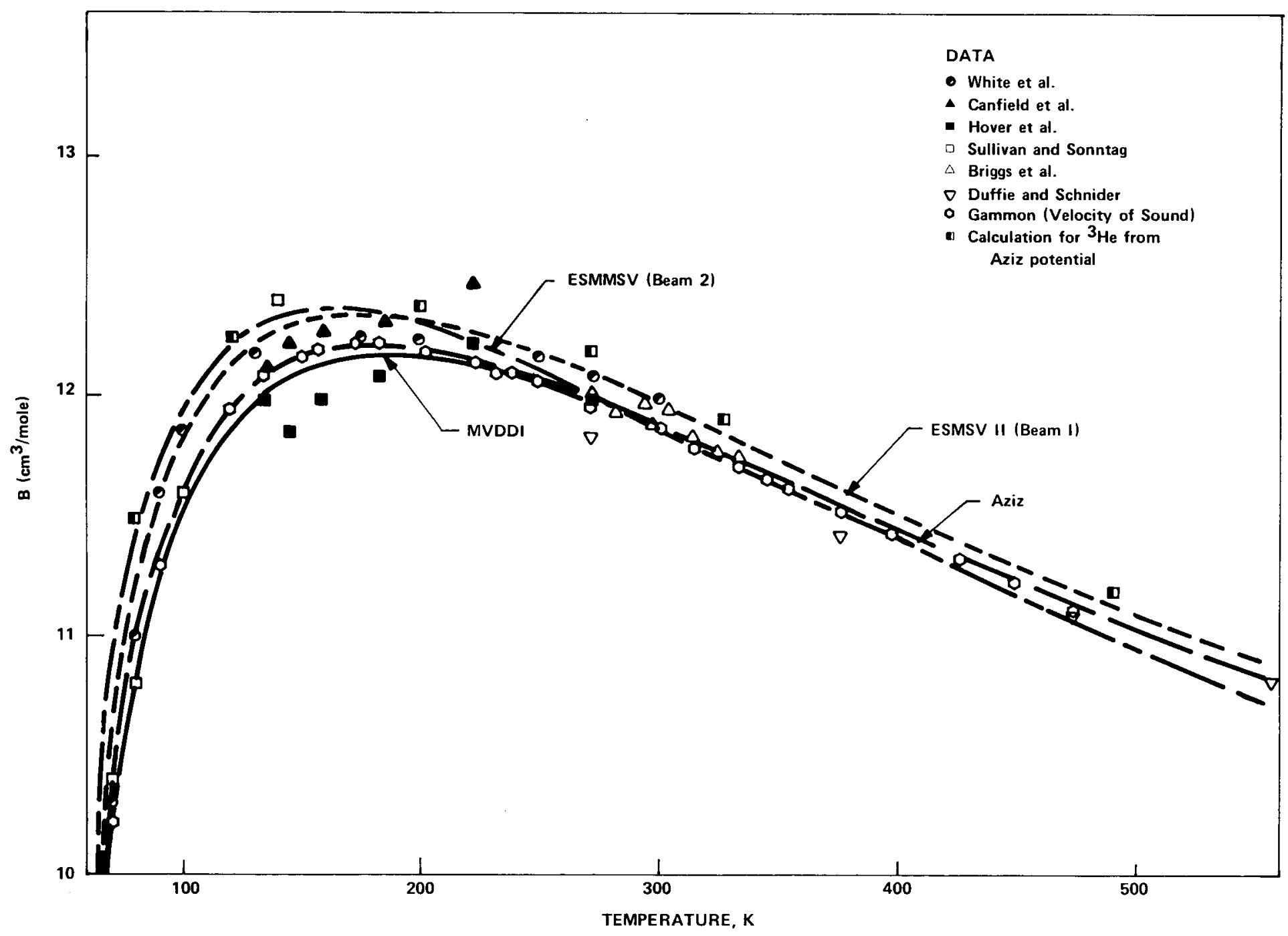

FIGURE 5 - Comparison of post-1950 second virial data with four recently proposed potential functions. 


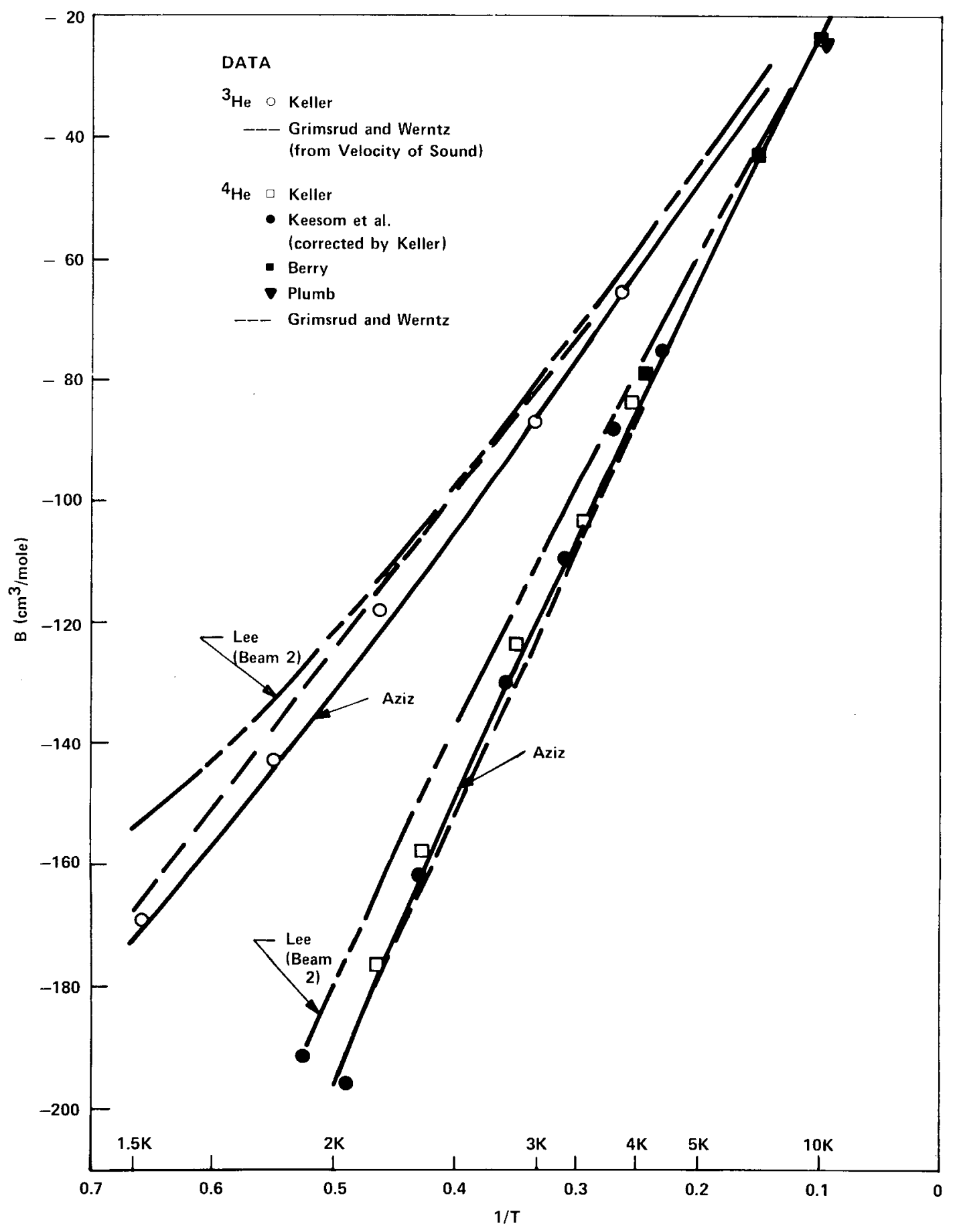

FIGURE 6 - Comparison of low-temperature second virial data for ${ }^{3} \mathrm{He}$ and ${ }^{4} \mathrm{He}$ with three recently proposed potential functions. 


\section{Metal Hydride Research}

\section{Pulse NMR Studies}

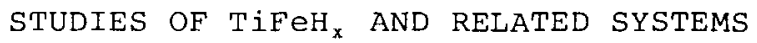

since TiFe and structurally related alloys are primary candidates for hydrogen storage applications, systematic investigations involving pulse nuclear magnetic resonance (NMR) are being conducted. Recent work at Mound Laboratory $[15,16]$ has shown that 1) electronic structures of the $\beta$ and $\gamma$ phases of $\mathrm{TiFeH}_{\mathrm{x}}$ are very different, 2) rigid lattice hydrogen interactions are very similar for these two phases, and 3 ) the hydrogen atoms are essentially immobile (i.e., diffusion constant $\left\langle 10^{-11} \mathrm{~cm}^{2} / \mathrm{sec}\right)$ below about 320 K. In unpublished work, Dr. G. C. Carter (National Bureau of Standards) and Dr. Y. Chabre (University of Grenoble) observed motional narrowing of the proton NMR spectrum above $\sim 350 \mathrm{~K}$ for a $B$-phase TiFeH, . sample and they deduced a diffusion activation energy of $0.3 \mathrm{eV}$ from their $\mathrm{T}_{2}^{\prime}$ spin-echo data. In light of the Carter-Chabre experiment, proton spin echo measurements have been performed at higher temperatures (i.e., between $300 \mathrm{~K}$ and $435 \mathrm{~K}$ ) on two predominantly $\beta$-phase samples and the $\gamma$-phase sample $\mathrm{Ti} \mathrm{i}_{1.04}$ $\mathrm{Fe}_{0.96} \mathrm{H}_{2} .0$ studied previously [16].

The results shown in Figure 7 confirm the onset of motional narrowing in $\beta$-phase TiFeH $x$ above $360 \mathrm{~K}$. An $\mathrm{E}_{\mathrm{A}}$ of $0.26 \mathrm{eV}$ was obtained for $\mathrm{Ti}_{0.9} \mathrm{Fe}_{1.02} \mathrm{H}_{1.0}$ and is in good agreement with the value $\sim 0.3 \mathrm{eV}$ determined by Carter and Chabre for $\mathrm{Ti}_{1.04}$

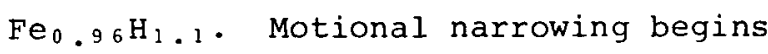
above $390 \mathrm{~K}$ in $\gamma$-phase $\mathrm{Ti}_{1} .04 \mathrm{Fe}_{0} .96 \mathrm{H}_{1} .98$, where a preliminary $E_{a}$ of $0.25 \mathrm{eV}$

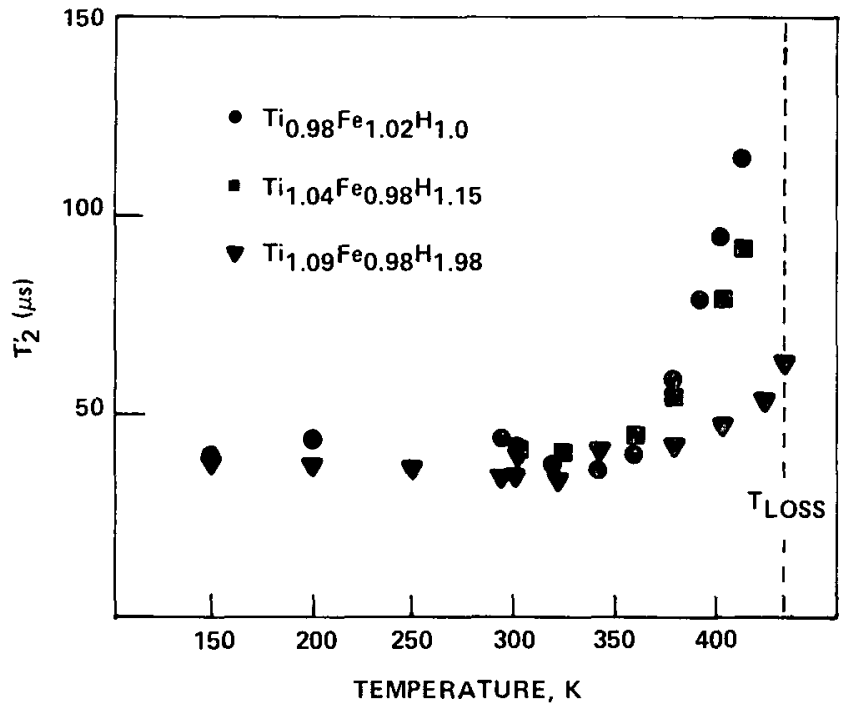

Figure 7 - Temperature dependence of the proton $T_{2}^{\prime}$ relaxation times for $\beta$ and $\gamma$ phases of $\mathrm{TiFeH}_{\mathrm{x}}$. Motional narrowing begins above $360 \mathrm{~K}$ in the $\beta$-phase and above $390 \mathrm{~K}$ in the $\gamma$-phase.

is obtained using the $\mathrm{T}_{2}$ data above $400 \mathrm{~K}$. This sample decomposed violently shortly after completion of the $\mathrm{T}_{2}$ measurements at $434 \mathrm{~K}$. Carter and Chabre observed a sudden hydrogen loss at $433 \mathrm{~K}$ with their $B$-phase sample. This sudden release of hydrogen gas at elevated temperatures is not surprising since the equilibrium dissociation pressure [17] for $\mathrm{TiFeH}_{\mathrm{x}}$ is above one atmosphere at room temperature. The hydride phases are stabilized [17] using air exposure at low temperatures to form a protective oxide layer which impedes the escape of hydrogen at elevated temperatures. However, when the thermal drive force for decomposition and the hydrogen permeation rate through the oxide surface film become sufficient, large quantities of hydrogen can be rapidly released.

Although the hydrogen mobility is slower in the $\gamma$-phase (i.e., motional narrowing starts at a higher temperature), the $E_{a}$ 
values are nearly equal for both $\beta$ and $\gamma$ phases. This suggests a similarity in the diffusion processes with a reduction of allowed jump-sites for the higher concentration $\gamma$-phase. Unfortunately, the phase diagram of the $\mathrm{TiFeH}_{\mathrm{x}}$ system at higher compositions and above $\sim 350 \mathrm{~K}$ is not well established [17]. It is possible that $\mathrm{TiFeH}_{2.0}$ undergoes a phase change near $390 \mathrm{~K}$ with a structure similar to the $\beta$-phase and an increased hydrogen mobility. Another possibility is the partial loss of hydrogen from the $\gamma$-phase $\mathrm{TiFeH}_{2}$.0 which results in the formation of the $\beta$-phase. Hence, more extensive studies are required before the diffusion mechanisms in the TiFeH $\mathrm{x}_{\mathrm{x}}$ phases can be deduced. Further NMR measurements on $\mathrm{TiFeH}_{x}$ and the related hydrides formed

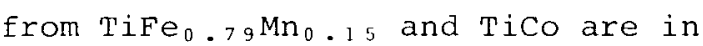
progress.

(Robert C. Bowman, Jx. and Albert Attalla)

DIFFUSION STUDIES IN $\mathrm{VH}_{\mathrm{x}}$

Among the more interesting unresolved problems in metal hydrides [18] are the mechanisms of hydrogen diffusion, including the roles of crystal structure, phase transformation, and isotope effects. The vanadium hydride system is a good candidate for experimental investigation; since several distinct phases are formed depending upon the hydrogen concentration and temperature, various factors affecting hydrogen diffusion can be systematically studied in a single hydride system. Consequently, an NMR study was initiated to evaluate proton diffusion behavior in $\mathrm{VH}_{\mathrm{x}}$. Previous results in this study were given in two prior reports in this series $[19,20]$ as well as in a recent publication [ 21$]$. However, these studies were limited to temperatures above $220 \mathrm{~K}$ due to experimental limitations. Since $V_{H}$ is known [22-26] to undergo phase transformations below $\sim 200 \mathrm{~K}$, extension of the NMR measurements to lower temperatures is desirable. Recent progress in the NMR study of hydrogen diffusion in $\beta$-phase $\mathrm{VH}_{x}$ is the subject of the present report.

The spin-lattice relaxation times $\left(\mathrm{T}_{1}\right)$ of protons were measured between $\sim 100 \mathrm{~K}$ and $2500 \mathrm{~K}$ using a 180-90-180 pulse technique. The majority of these measurements were performed at $34.5 \mathrm{MHz}$ although some data were also obtained at $23.3 \mathrm{MHz}$. The samples were always cooled from higher temperatures prior to the $\mathrm{T}_{1}$ measurements; a minimum waiting period of ten minutes ensured that equilibrium had been reached. Seven $V_{\mathrm{x}}$ samples with compositions between $\mathrm{VH}_{0} .39$ and $\mathrm{VH}_{0} .85$ were prepared from zone refined vanadium metal and high purity $\mathrm{H}_{2}$ gas. The NMR samples were sealed under vacuum in $7-\mathrm{mm}$ o.d. pyrex tubes.

The $\mathrm{T}_{2}$ values obtained at $34.5 \mathrm{MHz}$ are shown in Figure 8 (for $\mathrm{VH}_{0} .39, \mathrm{VH}_{0} .50$, $\mathrm{VH}_{0.70}$, and $\left.\mathrm{VH}_{0.85}\right)$ and Figure 9 ( $\mathrm{VH}_{0.53}$, $\mathrm{VH}_{0}, 6_{6}$, and $\left.\mathrm{VH}_{0}, 7_{4}\right)$. The major features of these data include:

1. A sharp break in the $T_{1}$ temperature dependence near $\sim 450 \mathrm{~K}$ due to a phase transition ;

2. Evidence for a low-temperature phase transition near or below $200 \mathrm{~K}$ in several $\mathrm{VH}_{\mathrm{X}}$ samples;

3. A strong composition-dependent minimum in the $T_{1}$ relaxation times indicating that hydrogen diffusion is the dominant spin relaxation mechanism [27] in $\beta$-phase $\mathrm{VH}_{\mathbf{x}}$ between $\sim 250 \mathrm{~K}$ and $450 \mathrm{~K}$. 


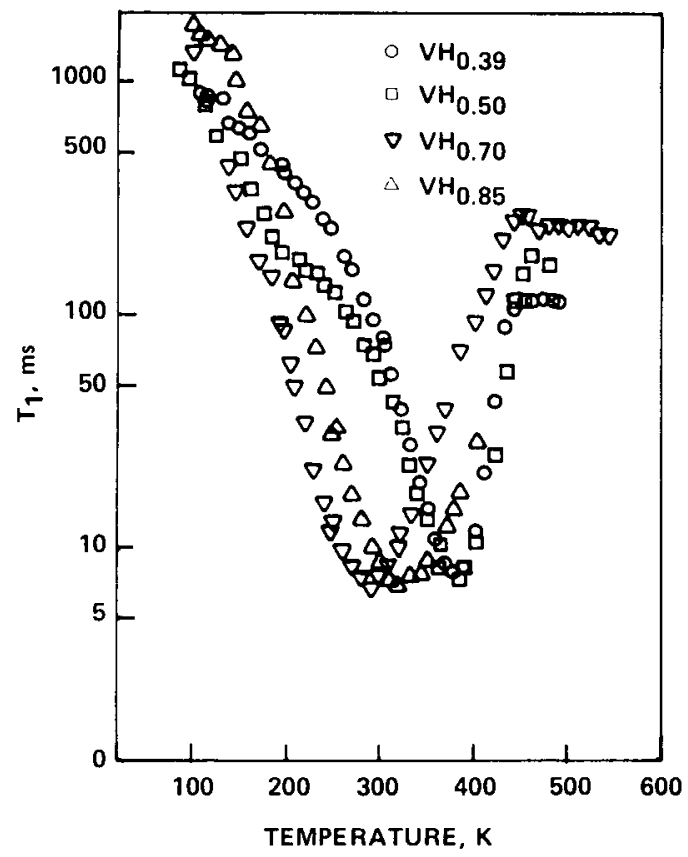

FIGURE 8 - Proton spin-lattice relaxation times $\left(T_{1}\right)$ in $V H_{x}$ as measured at $34.5 \mathrm{MHz}$.

Since the hydrogen mobility in $\alpha$-phase $\mathrm{VH}_{\mathrm{x}}$ is extremely rapid [18], diffusion does not contribute to $T_{1}$ relaxation in this phase and cannot be evaluated from the present data. Furthermore, the phase transformation near $200 \mathrm{~K}$ complicates the analysis since discontinuities in the $T_{1}$ temperature dependence are observed. However, a tentative evaluation of the hydrogen diffusion behavior in $\beta$-phase $\mathrm{VH}_{\mathrm{x}}$ has been performed using the model described below.

The application of NMR to diffusion in metal hydrides was recently reviewed by cotts [27]. Generally two major factors contribute to the $\mathrm{T}_{1}$ for hydrogen nuclei. They obey

$$
\mathrm{T}_{1}^{-1}=\mathrm{T}_{1 \mathrm{~d}}^{-1}+-\mathrm{T}_{1 \mathrm{e}^{-1}}
$$

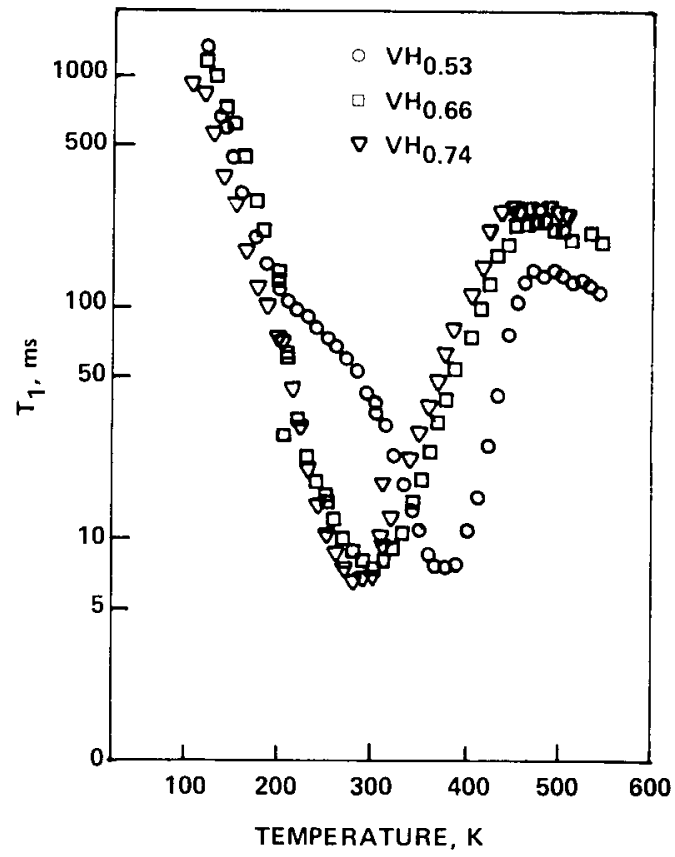

FIGURE 9 - Additional proton spin-lattice relaxation times $\left(T_{1}\right)$ in $V H_{x}$ as measured at $34.5 \mathrm{MHz}$.

where $T_{1 d}$ is related to the diffusion correlation time, $\tau_{c}$, and $T_{1 e}$ is caused by the contact interaction between hydrogen nuclei and the conduction electrons. $T_{1}$ e obeys the Korringa relation

$$
T_{1} \in T=K
$$

where $T$ is the absolute temperature and $K$ is a constant related to the density of states at the Fermi surface. Since the $\mathrm{V}-\mathrm{H}$ dipolar interactions dominate [28] $\mathrm{T}_{1} d$ in any $\mathrm{VH}_{x}$ phase, the $\mathrm{H}-\mathrm{H}$ dipolar contributions can be neglected during analysis for $\tau_{0}$. Using the BPP model [27] to deduce $\tau_{c}$ from the $T_{I}$ data, we obtain 
$1.491 \mathrm{~T}_{1}$

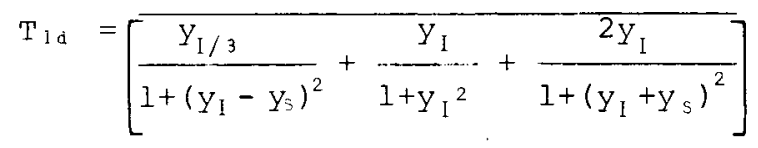

where $y_{I}=1 / \omega_{I} \tau_{c}, y_{s}=1 / \omega_{s} \tau_{c}, \omega_{I}$ is the proton resonance frequency, $\omega_{s}$ is the vanadium resonance frequency, and $T_{1 m i n}$ is the minimum value of $T_{1 d}$. After correcting the $T_{1}$ data in Figures 8 and 9 for the $T_{1}$ e contributions, which were estimated from the $T_{1}$ values at low temperatures where $\mathrm{T}_{1 \text { d }}$ does not contribute, Equation 3 was solved to determine the $\tau_{c}$ values for each $V_{\mathrm{x}}$ sample. The temperature dependences of $\tau_{c}$ for $\mathrm{VH}_{0} .39$, $\mathrm{VH}_{0} .5, \mathrm{VH}_{0} .70$, and $\mathrm{VH}_{0} .85$ are shown in Figure 10. Similar results were obtained for the other $\mathrm{VH}_{x}$ samples.

The correlation times for diffusion processes normally exhibit [27] Arrhenius behavior and follow

$$
\tau_{\mathrm{c}}=\tau_{\infty} \exp \left(\mathrm{E}_{\mathrm{a}} / \mathrm{kT}\right)
$$

where $E_{a}$ is the diffusion activation. However, the $\tau_{\circ}$ values deduced from $\mathrm{T}_{1}$ for $V_{H}$ indicate that different results for $\mathrm{E}_{\mathrm{a}}$ are obtained above and below $\mathrm{T}$ imin. These energies are shown in Figure 11 for the seven $\mathrm{VH}_{\mathrm{x}}$ compositions. Significant decrease of $E_{a}$ is observed as $x$ increases, although an apparent minimum occurs near $\mathrm{VH}_{0.7}$ for the $\mathrm{E}_{\mathrm{a}}$ below the $\mathrm{T}_{1 \mathrm{an}}$.

Since the $\mathrm{V}-\mathrm{H}$ interactions dominate $\mathrm{T}_{1 \mathrm{~d}}$, the $\mathrm{T}_{1}$ minimum occurs when

$$
\omega_{1} \tau_{c}=0.922
$$

The temperatures at which $T_{1 m 1 n}$ is found for each $\mathrm{VH}_{\mathrm{x}}$ sample are summarized in

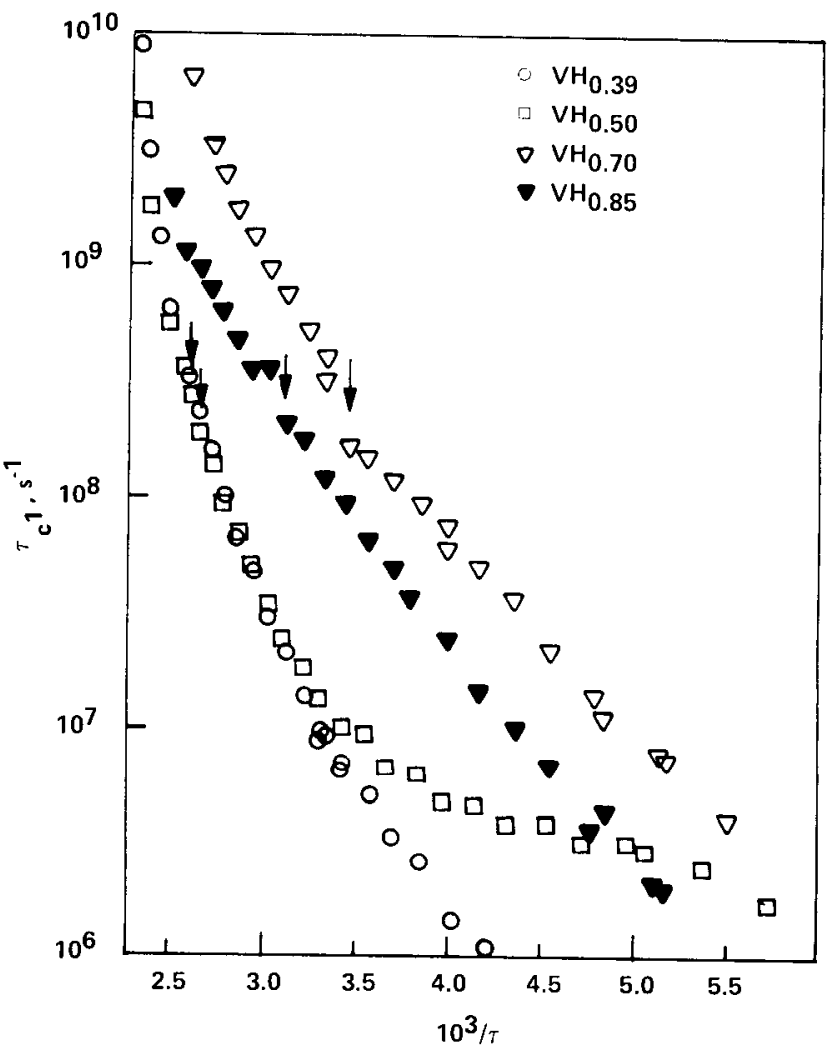

FIGURE 10 - Temperature dependence of diffusion correlation times $\left(\tau_{c}\right)$ as calculated with BPP model using proton $T_{1}$ data corrected for conduction electron interactions.

Figure 12. Timin show a strong dependence on hydride stoichiometry with a minimum near $\mathrm{VH}_{0.75}$. The hydrogen diffusion constant, D, obeys

$$
D=\frac{\left.1<\lambda^{2}\right\rangle}{6} \frac{\tau_{C}}{\tau_{C}}
$$

where $\left\langle\lambda^{2}\right\rangle$ is the mean squared hydrogen jump distance. Therefore, the temperature dependence for $T_{1 m 1 n}$ implies the hydrogen mobility in $\beta$-phase $\mathrm{VH}_{\mathrm{x}}$ rapidly increases above $\mathrm{VH}_{0} .5$, reaches a maximum rate near $\mathrm{VH}_{0.75}$, and decreases for higher stoichiometries. 


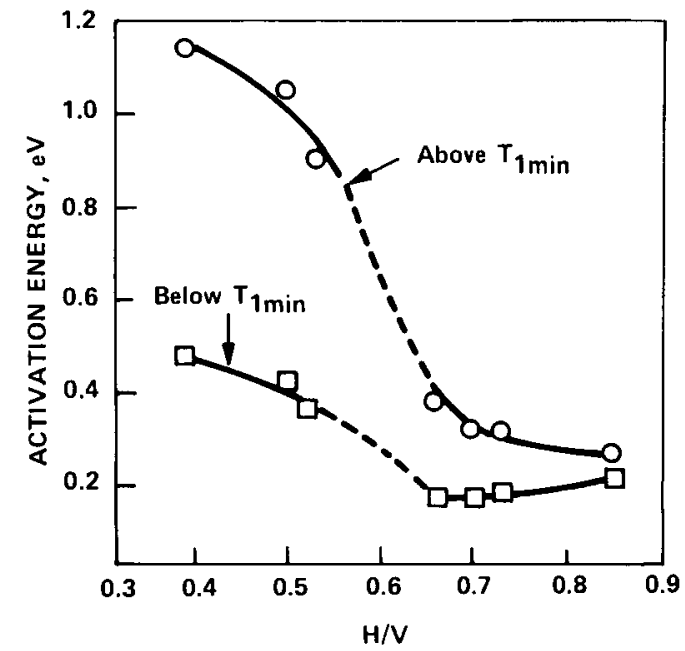

FIGURE 11 - Activation energies ( $E_{a}$ ) for hydrogen diffusion in $B$-phase $V H_{x}$.

The two major features of hydrogen diffusion behavior in $\beta$-phase $V_{\mathbf{x}}$ deduced from the present $T_{1}$ measurements are:

1. $E_{a}$ is concentration dependent and becomes smaller as the hydrogen content in the $B$-phase increases.

2. D has a maximum value near $V_{0.75}$ and decreases at lower and higher stoichiometries.

Both of these observations are inconsistent with the hydrogen sublattice vacancy model [29] which describes hydrogen mobilities in the face-centered cubic $\mathrm{PdH}_{1-y}[30]$ and $\mathrm{TiH}_{2-\mathrm{y}}[31,32]$. The diffusion constant obeys

$$
D=y v_{0} \exp \left(-E_{a} / k T\right)
$$

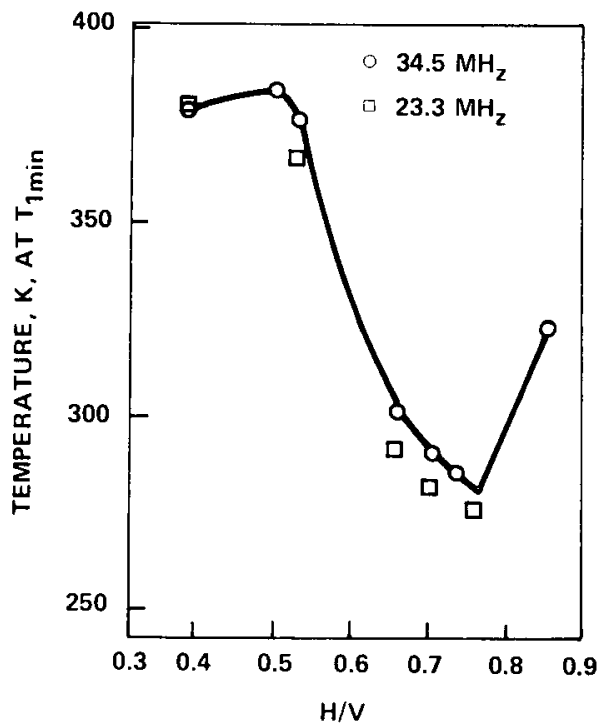

FIGURE 12 - Dependence of spin-lattice relaxation times minima on hydrogen content in $\beta$-phase $V H_{x}$.

for the vacancy model, where $y$ is the concentration of hydrogen vacancies, vo is the diffusion attempt frequency, and $E_{a}$ is independent of vacancy concentration. Hence, Equation 7 predicts D would decrease as the hydrogen content increases in $\beta$-phase $\mathrm{VH}_{\mathrm{x}}$. Since the results in Figure 12 disagree with this prediction, the vacancy model apparently does not represent hydrogen mobility in this system.

An alternative model for hydrogen diffusion in B-phase $\mathrm{VH}_{x}$ is presented. When $x<0.5$, only the hydrogen sites corresponding to the $\mathrm{V}_{2} \mathrm{H}$ superlattice $[24$, 25] are occupied and mobility is restricted. However, as the hydrogen content increases, the other allowable 
octahedral sites in $\beta-\mathrm{VH}_{\mathrm{x}}$ become partially occupied, lowering the activation energy by providing additional diffusion paths for the hydrogen atoms. When the stoichiometry is higher than $\mathrm{VH}_{0} .75$, the concentration of these unfilled sites starts to decrease. This should lead to a vacancy type of mechanism with a nearly constant $E_{a}$. The rapid increase in the $\mathrm{T}_{1 \mathrm{~m}} \mathrm{n}$ temperature for $\mathrm{VH}_{0} .8 \mathrm{~s}$ is consistent with the vacancy mechanism at higher concentrations. Although this model provides a qualitative description of diffusion in $\mathrm{VH}_{\mathrm{x}}$, detailed analysis is currently not possible because of the low and high temperature phase transitions. Without a better understanding of the structures of these phases their influence on hydrogen mobility cannot be evaluated.

The occurrence of two activation energies has been observed in the structurally related hydrides $\mathrm{TaH}_{\mathbf{x}}$ [33] and $\mathrm{NbH}_{\mathrm{x}}$ [34]. In these cases the $y-\beta$ phase transition is responsible for a smaller activation energy above the transition temperature. However, the larger $\mathrm{E}_{\mathrm{a}}$ occurs above $T_{1 m i n}$ for $V_{x}, V_{x}$ being single phase in this temperature region. Consequently, the $\alpha-\beta$ phase change is not responsible. Very similar behavior was found by Rohy

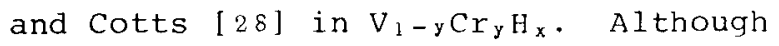
the BPP model used to determine $\tau_{c}$ from $T$, becomes inadequate [35] at temperatures significantly below $\mathrm{T}_{\mathrm{Imi}}$, this effect should not occur near $\mathrm{T}_{1 \mathrm{~min}}$. It is evident that additional studies are required before the differences in $\mathrm{E}_{a}$ values obtained from $T_{1}$ measurements can be adequately explained. These studies are now in progress.

(Robert C. Bowman, Jr., Albert Attalla, and Bartlett $D$. Craft)

\section{Electrical Resistivity Studies of Phase Transformations in $\mathrm{VHx}$}

Although vanadium hydride does not have ideal properties for storage purposes, it is a good representative of metal hydrides and has a phase diagram and properties of considerable scientific interest. The phase diagram has been under study for some years now, but is not yet fully understood. Above room temperature, the basic phase diagram was first proposed by Maeland [36] in 1964 as a result of $\mathrm{X}$-ray diffraction data which indicated crystal structure and changes in the lattice parameters. In 1969, Westlake [37] discovered, by means of electrical resistivity measurements, a low temperature order-disorder type of transformation in the composition range of $\mathrm{VH}_{0.5}$ to $\mathrm{VH}_{0.7}$. Fukai and Kazama [38] have produced data from both electrical resistivity and nuclear magnetic resonance while Asano [24] and his co-workers showed a slightly different high-temperature phase boundary as a result of calorimetric measurements.

A composite in Figure 13 shows the phase diagram as presently understood. The region from about $\mathrm{VH}_{0} \cdot 4$ to $\mathrm{VH}_{0} \cdot 8$ is particularly uncertain, and is the subject of this investigation. As far as is presently known, the b.c.c. $\alpha$-phase is simply an interstitial solution of hydrogen predominantly occupying tetrahedral sites at random, whereas the b.c.t. $\beta$-phase is an ordered phase. The region between the two high-temperature boundaries was originally referred to as a mixture of $\alpha$ and $\beta$, but results of the NMR and calorimetry experiments suggest it 


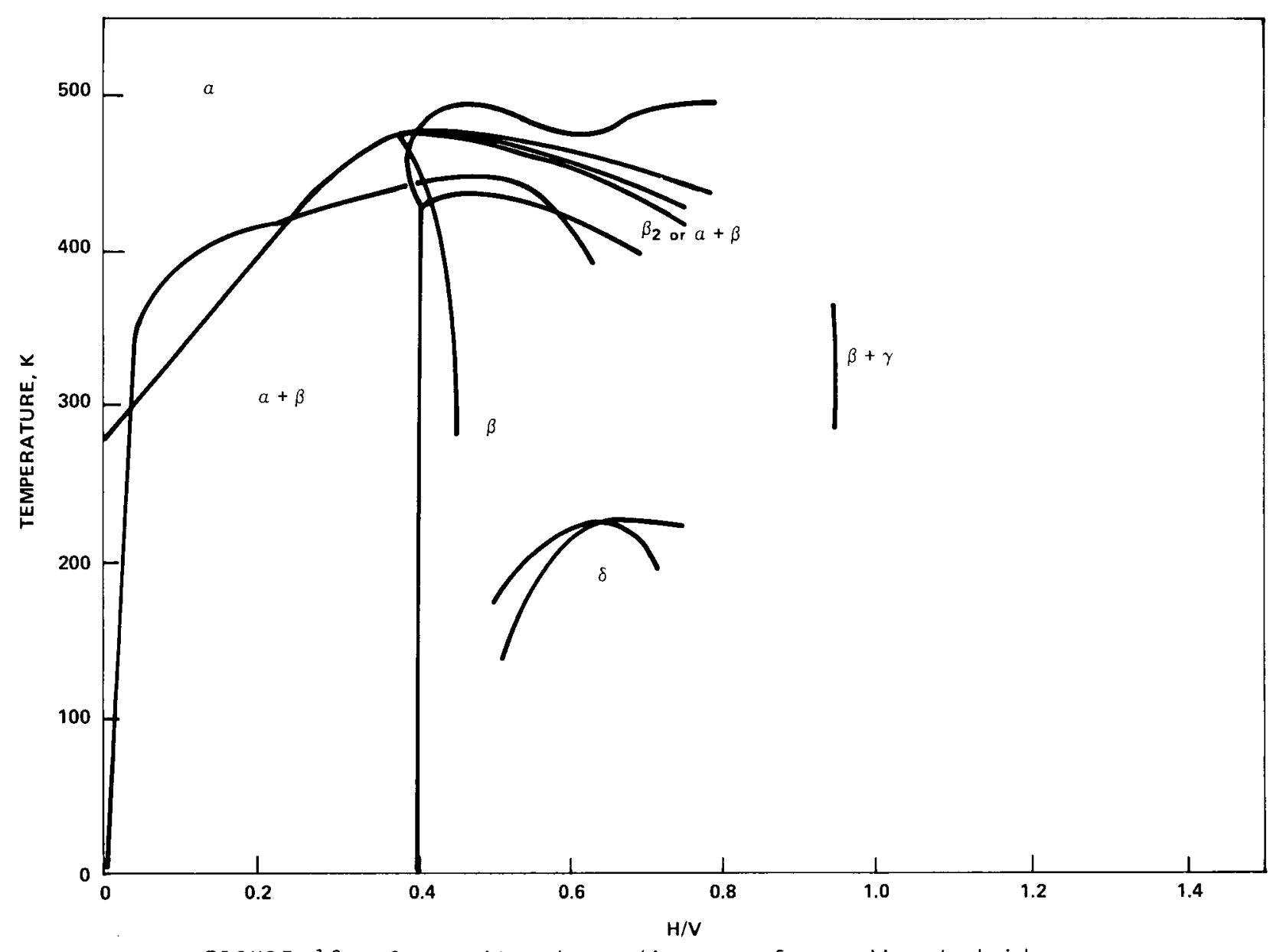

FIGURE 13 - Composite phase diagram of vanadium hydride.

may be a single $\beta_{2}$ phase. Beyond $\mathrm{VH}_{0}, 9$, an f.C.C. $\gamma$-phase of composition $\mathrm{VH}_{2}$ begins to appear.

In order to better determine the exact points of the phase boundaries, we have begun an investigation based upon electrical resistivity measurements. Because of the brittleness of the hydride product, pieces of vanadium foil are first cut to appropriate dimensions for resistivity measurements. The vanadium foil is then placed in a furnace and outgassed under vacuum at $973 \mathrm{~K}$. After several exposures to hydrogen gas at this temperature, the vanadium, now activated, is cooled to $673 \mathrm{~K}$ and a measured amount of hydrogen is added. Usually about $98 \%$ to $99 \%$ is absorbed as the hydride cools to room temperature. Measuring the final pressure allows exact determination of the composition. The composition is then confirmed by measuring the increase in mass of the foil and by analyzing the volume of hydrogen gas evolved through thermal decomposition. Four compositions have been synthesized and studied so far: $\mathrm{VH}_{\mathrm{x}}$ with $\mathrm{x}=0.43,0.53,0.60$, and 0.80 .

Resistivity measurements are made using ac techniques with a lock-in amplifier and a four-point probe. The sample is contained in a cryostat to allow both low- and high-temperature measurements. 
The range of $100 \mathrm{~K}$ to $500 \mathrm{~K}$ covers all three transitions for the compositions studied. Hydrogen loss at the higher temperature region is a possible problem, and although it does not appear to happen until well beyond the highest temperature phase transition, we plan to monitor the pressure in future work to detect any release of hydrogen.

Resistivity is plotted against temperature in Figure 14. The plot for $\mathrm{VH}_{0.60}$ shows all three phase transformation points, which appear as changes in the p-T slope. The straight line is that of pure vanadium. The two lowest compositions studied, 0.43 and 0.53 , do not show the prominent lowtemperature drop-off, although it is questionably visible on a larger scale for $V_{0} .53$ at $171 \mathrm{~K}$. The high temperature points are much more pronounced, though, for these compositions. These three compositions are all in general agreement with Westlake's data. They show a general trend in that the residual resistivity increases with the addition of hydrogen at low temperatures, but the absolute resistivity is seen to drop at $500 \mathrm{~K}$ with the increase of hydrogen. - Our $\mathrm{VH}_{0.80}$ sample continues this pattern and shows

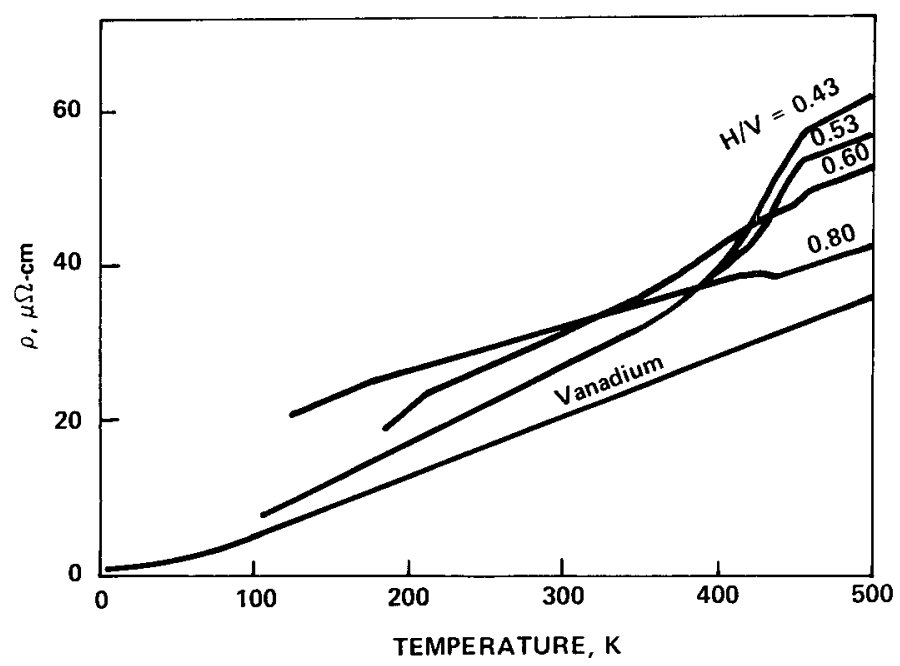

two high-temperature transitions, which, as far as we know, have not been reported near this composition.

The high-temperature phase changes for 0.43 and 0.53 are indicated by two sharp and distinct points which exhibit hysteresis upon cooling. The $\mathrm{VH}_{0} .60$ has no sharp point for the high-temperature transition. The $\mathrm{VH}_{0} .80$ differs even more. In it, the first high-temperature phase transformation shows up as an increase in slope -- all other compositions showed a decrease after that point. The highest temperature transition is indicated by a sudden drop in the curve, which is also unlike the other samples.

The data show another interesting feature near $\mathrm{x}=0.60$ when resistivity is plotted against composition. Since the purpose of this research is to detect phase changes by noting changes in resistivity with respect to temperature, exact values of $\rho$ are not as important as is the sensitivity. However, absolute resistivity vs. composition has been plotted, and even after considering experimental error, shows a sudden

FIGURE 14 - Resistivity plots of various hydride compositions show change in slope not present in pure vanadium. 
change in resistivity near a composition of 0.60 . When such data are plotted for several different temperatures, as in Figure 15, all lines begin to converge at $\mathrm{VH}_{0.60}$ and all lines seem to intersect at $\mathrm{VH}_{1} .0$, indicating that the latter composition would have temperature independent resistivity of about 32 $\mu \Omega-\mathrm{cm}$. More accurate resistivity measurements on more samples will hopefully verify this.

After completing measurements on the vanadium-hydrogen system, we also plan to continue with the vanadium-deuterium system. Apparently no resistivity measurements have yet been reported on this deuteride. The phase diagram, although studied only to a limited

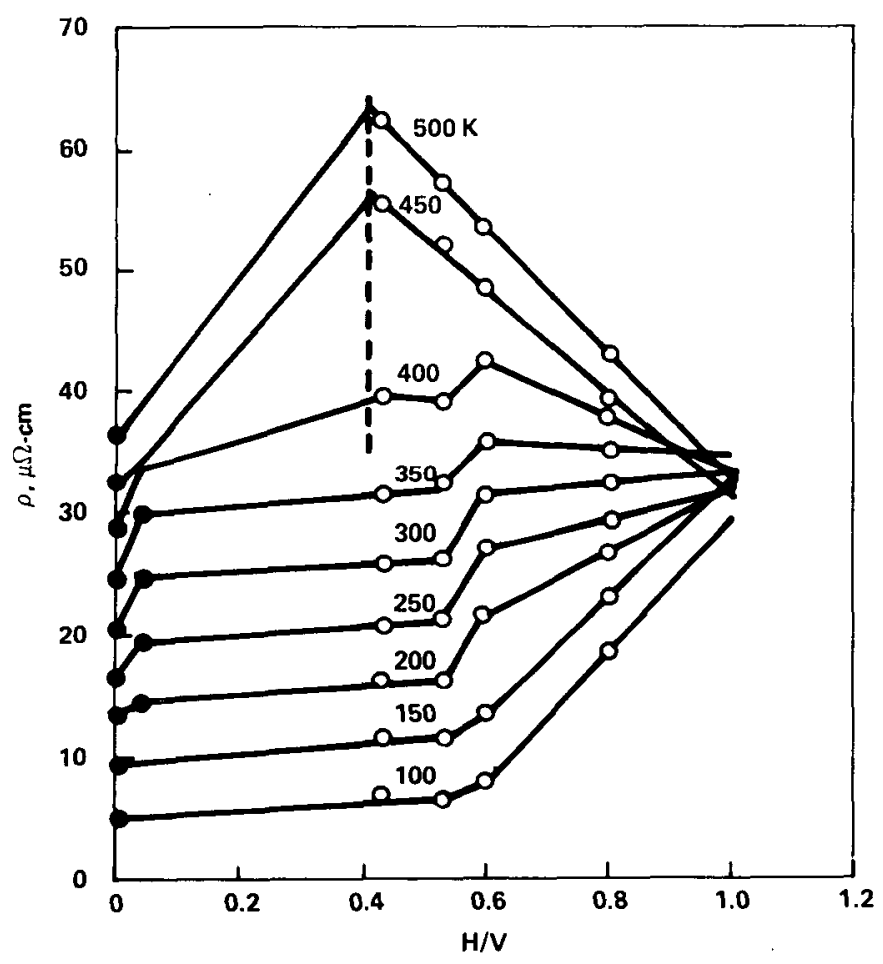

FIGURE 15 - Resistivity plots at various temperatures show possible intersection at $\mathrm{VH}_{1,0}$. extent, seems to differ from that of the hydride much more than other metal deuterides differ from their corresponding hydrides. We also plan to study the vanadium-tritium system using the same methods. Virtually no work has been done in this area, and the great

differences between the deuteride and the hydride phase diagrams imply that the tritide work will be of extreme interest. (Michael W. Pershing, J. F. Thomas, Ir., Robert $C$. Bowman. Ir., and Calvin M. Lovel

\section{Electron Paramagnetic Resonance Studies of Transition Metal Ions in Lithium Hydride}

Electron paramagnetic resonance (EPR)

studies of transition metal and rare earth ions in diamagnetic metal hydrides have been initiated to obtain a better understanding of metal-hydrogen chemical bonding. Trends in the covalent contribution to the meta1-hydrogen bonds are investigated by analyzing magnetic g-factors and hyperfine constants of paramagnetic impurity ions in binary and ternary hydrides whose bonding characteristics vary from ionic to metallic.

RHODIUM IONS IN LIH AND LID SINGLE CRYSTALS

In previous reports, we have described EPR spectra in $L i H$ and $L i D$ single crystals, arising from two distinct paramagnetic $\mathrm{Rh}^{+}{ }_{2}$ species, $\mathrm{A}$ and $\mathrm{B}$. Due to line-broadening, species $A$ is not observed above $200 \mathrm{~K}$, allowing a complete and detailed spectral analysis of species $B$. The details and conclusions of that analysis are briefly described in the most recent progress report [39] and have been published in Physics Letters [40].

A careful study of species A requires liquid nitrogen temperature and high microwave power (since $B$ saturates more readily than $A$, 
high power enhances the A spectra). Unfortunately, experimental difficulties (e.g., Iiquid $\mathrm{N}_{2}$ bubbling, lack of a suitable goniometer mount for use with the liquid $\mathrm{N}_{2}$ Dewar) had prevented a detailed analysis of $A$. These difficulties have now been overcome. In addition, the passage of time has significantly enhanced the A spectra, apparently because B is less stable than $A$ with respect to room-temperature annealing following ultraviolet irradiation.

We now have a complete study of the orientation dependence of the A spectra for $\mathrm{Rh}^{+}$ ions in single crystal lithium hydride. Figure 16 shows first derivative spectra of species $A$ in $L i D$ and $L i H$, respectively. The outstanding feature is the superhyperfine structure (shs) appearing in the LiH spectra (the protium magnetic moment is much larger than that for deuterium). Analysis shows that $A$ is a nearly axial species with $g_{x}=2.07, g_{y}=2.06$ and $g_{z}=2.005$. Since $g_{1}\left(g_{x} \simeq g_{y}\right)>g_{11} \simeq g_{e}$ the paramagnetic electron belongs to the

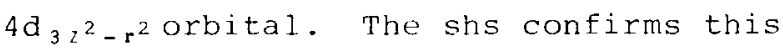
interpretation and the total magnetic spin density on the axial (z) ligands is found to be $9 \%$. It is interesting to compare these results with the results obtained for species $B$, for which $g_{11}(2.11)>$ $g_{1}(2.04)>g_{e}$, implicating the $4 \mathrm{~d}_{\mathrm{x}^{2}-\mathrm{y}^{2}}$ orbital (confirmed by the shs). In the latter case, the total magnetic spin density on the planar $(x, y)$ ligands is $6 \%$.

The experimental work on rhodium ions in lithium hydride has essentially been completed. Some additional analysis is required. The parameters obtained in this work that measure covalency effects should be useful for correlation with similar parameters in analogous ternary hydrides, such as $\mathrm{Li}_{4} \mathrm{RhH}_{5}$.

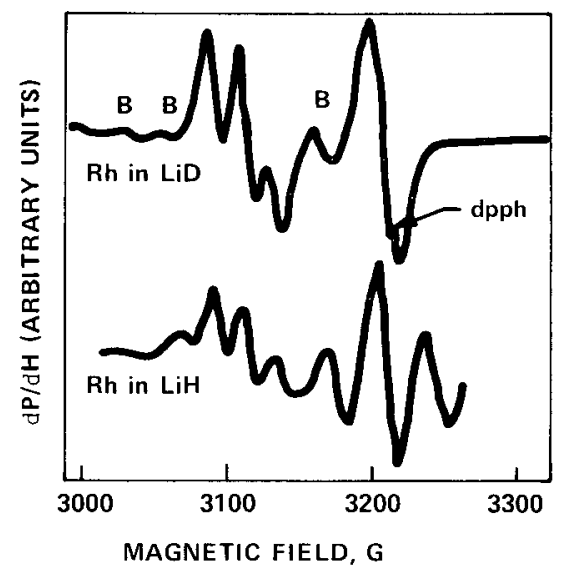

FIGURE 16 - First-derivative X-band EPR spectra for species $A$ of $R h^{+2}$ in $L i D$ and LiH single crystals. The crystals were irradiated with ultraviolet light at room temperature and ohserved at $77 \mathrm{~K}$ and high microwave power $(150 \mathrm{~mW})$. The magnetic field is along a [100] direction. Adsorptions belonging to species $B$ are indicated.

NICKEL IONS IN LIH SINGLE CRYSTALS

We have completed an orientation study at $77 \mathrm{~K}$ of the EPR spectra of LiH containing nickel ions as an impurity $(\approx 10-100$ ppm). We have strong evidence for two species--one shows axial symmetry with $g_{11}=2.21, g_{1}=2.06$, and $g_{11}>g_{1}>g_{e}$

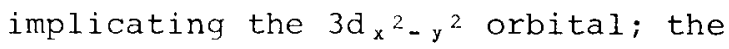
other is isotropic $\left(g_{150}=2.09\right)$ and is probably a Jahn-Teller ion. Both species show anistropic shs, and are most likely $3 \mathrm{~d}^{9}(\mathrm{~S}=1 / 2) \mathrm{Ni}^{+}$complexes. We are now analyzing these data. Preliminary interpretation shows that the covalency effect is appreciably greater for the nickel complexes than for the rhodium complexes. (George C. Abe11)

\section{Band Theory and Electronic Structure}

Implementation of the computer codes required for a molecular quantum mechanical study of interstitial metal hydrides is now proceeding smoothly. These computer 
programs are all being set up on Brookhaven's 6600 and 7600 CDC computers. Initially, severe difficulties were encountered in establishing a reliable communications link between our Tektronix 4051. programmable terminal and Brookhaven's time-sharing system because of idiosyncrasies associated with CDC's INTERCOM system and poor-quality FTS phone lines. However, these difficulties have now been overcome with the development of failsafe 4051 programs which take these factors into account.

In addition to the above codes for sending and receiving data, several other programs of general utility have been designed for the 4051 . A general initiating program has been written which automatically establishes the environmental parameters appropriate for various tasks and which links all the other 4051 codes into an easy-to-use coherent whole. A program for editing tape files has been developed; it permits convenient local development of FORTRAN source programs and allows for local maripulation of input and output data sets stored on the magnetic tape cartridges of the terminal. Utility programs for copying and auditing these tape data files have also been written. In addition, oneand two-dimensional function plotting programs have been designed which can plot data generated locally or incoming from an external host computer. For the most part, the 4051 programs have been developed as a prerequisite to convenient and efficient implementation of the large molecular quantum mechanical computer codes being set up on Brookhaven's computers.

Aside from various minor utility programs, six major programs have thus far been implemented and tested. These programs include an improved BIGMOLI integrals labels program; the BIGMOLI integrals generation program; and a revised program for converting a raw BIGMOLI integrals file to a standard POLYATOM-type integrals file. Since all the electronic structure codes are of the usual basis set-expansion (LCAO) type, these three programs represent the basic starting point for any computational theoretical study, providing the 1- and 2-electron integrals required as input by all the other programs. Of the various self-consistent field wavefunction (SCF) programs in our possession only the unrestricted Hartree-Fock (UHF) program has been fully implemented and tested thus far. This program represents a significant improvement over the older version; in fact, the design revisions will serve as a guide to analogous improvements in other more complicated SCF programs as they are set up. Finally, one- and two-dimensional orbital amplitude evaluation programs have been written and tested. These programs are fully compatible with the corresponding 4051 plotting programs mentioned earlier and will be used to provide visual representations of the results obtained from our calculations.

Currently, work is under way to implement a revised version of our open-shell restricted Hartree-Fock (RHF)/restricted Generalized Valence Bond (GVB) program. Since this SCF program figures predominantly in our future theoretical studies, an effort is being made to make this program as efficient and easy to use as possible. Work is also proceeding on a configuration interaction (CI) program along with the concurrently required integral manipulation and transformation programs. They will make it possible to check on the validity of SCF results and 
also to obtain more quantitative results than is possible at an SCF level. Finally, work is under way in converting a recently obtained potentials program to our use so that calculations not otherwise possible at an all-electron level can be performed on systems involving moderately heavy metal atoms.

Once these computer codes have been imple-mented and thoroughly tested, it is planned to perform calculations on prototype metal and metal hydride clusters such as lithium and lithium hydride to fully evaluate our computational capabilities. Developing the necessary experience and expertise with such cluster calculations will permit us to proceed to more complicated systems, such as intermetallic hydrides involving titanium and the first-row transition metals (i.e., TiNi, TiCu, TiFe, and TiCo). (Frank w. Bobrowicz) 


\section{Separation Chemistry}

\section{Problems in Plutonium Chemistry (V): The Solubility of Tetravalent Plutonium Hydroxide}

An unresolved question in plutonium chemistry is which numerical value best represents the solubility product of tetravalent plutonium hydroxide. Even though the technique of assigning a particular number as the value of the solubility product of a particular hydrous oxide is often criticized [41], it is nevertheless sometimes convenient to use such a number to demonstrate typical or possible solubilities. Widely divergent values for the solubility product of tetravalent plutonium hydroxide have been experimentally determined. A chemical reaction often overlooked may help to partially explain some of the discrepancy.

As the $\mathrm{pH}$ of a tetravalent plutonium solution increases, the solubility of the plutonium would decrease if the solubility were accurately represented by Equation I only

$$
\mathrm{Pu}^{4+}+4 \mathrm{HOH}=\mathrm{Pu}(\mathrm{OH})_{4}+4 \mathrm{H}^{+}
$$

But, the solubility of the plutonium may actually be increased by the formation of various hydroxy complexes of tetravalent plutonium. Failure to account for these complexes may be a potential source of error in experimental determination of the solubility product of tetravalent plutonium hydroxide. Failure to include hydroxy complexes is not the only potential source of error, however, as disproportionation products derived from tetravalent pluto- nium may remain in the solution and affect the solubility product.

Suspensions of tetravalent plutonium hydroxide display some oxidation-reduction potential, which may derive from a variety of sources, such as impurities in the solution, radiation effects, or dissolved oxygen. This potential may act upon the hydrous plutonium oxide as in Equation 2

$$
\mathrm{Pu}(\mathrm{OH})_{4}=\mathrm{PuO}_{2}^{+}+2 \mathrm{HOH}+\mathrm{e}
$$

Equation 2 is not dependent upon acidity, and, at constant potential, the pentavalent plutonium derived from oxidation of the tetravalent plutonium hydrous oxide may be an appreciable proportion of the soluble plutonium in equilibrium with $\mathrm{Pu}(\mathrm{OH})_{4}$. Such pentavalent plutonium may lead to spurious results in experimental determinations of the solubility product of tetravalent plutonium hydroxide. Since the extent of the oxidation reaction cannot be determined in advance, and may be highly variable, it is difficult to predict how much error in solubility product determinations may be introduced by the oxidation reaction. (The redox potential of a suspension of the hydrous oxide may also be related to the $\mathrm{pH}$ of the suspension.) The presence of unsuspected concentrations of pentavalent plutonium in the presence of the hydrous oxide may help explain the discrepancies so often observed in measuring. the solubility product of this hydrous oxide [42].

Table 2 suggests the percentages of pentavalent plutonium in equilibrium with hydrous tetravalent plutonium oxide at various $\mathrm{pH}$ values. As the $\mathrm{pH}$ increases, pentavalent plutonium becomes the principal species among the soluble valence states. 
The following values were assumed for

Table 2: potential $=+0.4 \mathrm{~V}$ Vs N.H.E.; all plutonium ion alpha coefficients $=1$; $\mathrm{K}_{1}=6.97 \mathrm{E}-04 ; \mathrm{K}_{2}=13.2 ;$ standard potential of $\mathrm{PuO}_{2}^{2+} / \mathrm{PuO}_{2}^{+}$couple $=0.9164 \mathrm{~V}$.

-Table 2 - PERCENTAgE OF PENTAVALENT PLUTONIUM AMONG SOLUBLE PLUTONIUM IONS IN EQUILIBRIUM WITH TETRAVALENT PLUTONIUM HYDROXIDE*

$\begin{array}{cl}\frac{\mathrm{pH}}{5} & \frac{\% \mathrm{pu}^{+}}{0.14} \\ 6 & 93.3 \\ 7 & 99+ \\ 8 & 99+\end{array}$

* Potential is constant at $+0.4 \mathrm{~V}$.

The solubility of tetravalent plutonium hydroxide is also affected by the alpha coefficients of the four plutonium oxidation states which may be in equilibrium with the hydroxide. These alpha coefficients are probably subject to empirical approximation in a variety of ways. For example, if a low solubility neodymium salt were allowed to equilibrate in a liter of pure water (containing an inert salt to adjust the ionic strength of the solution) and similarly allowed to equilibrate in a liter of natural water (as taken from the environment), two differing solubility measurements are likely to result. The first measurement, taken on the pure water, is an indication of the intrinsic, or ionic, solubility of the neodymium salt. The second measurement, taken on the sample of environmental water, represents this intrinsic solubility plus any additional solubility due to complexation of the neodymium by naturally occurring complexing agents. The neodymium alpha coefficient is the ratio of the total neodymium concentration in a solution divided by the concentration of uncomplexed, ionic neodymium. Hence, mere division of the neodymium solubility in the natural water by the neodymium solubility in the pure water is an approximation of the neodymium alpha coefficient in the natural water [43]. Since the trivalent neodymium ion is similar to the trivalent plutonium ion, this method may yield an approximation of the alpha coefficient for trivalent plutonium in a sample of natural water. This approximation may be sufficiently accurate for the purposes of valence state distribution computations [44]. Similar experiments with tetravalent thorium, petavalent neptunium and hexavalent uranium may yield approximations for the alpha coefficients for tetravalent, pentavalent, and hexavalent plutonium.

The determination of chemical speciation with computer models based upon stability or formation constants [45] has recently been criticized [46]. The determination of oxidation state distributions by use of empirically determined alpha coefficients [43] would appear to be immune, however, to some of these criticisms.

(Gary L. Silver)

\section{Problems in Plutonium Chemistry (VI): Kinetics of Plutonium Disproportion- ation Reactions}

Considerable interest in the kinetics of plutonium reactions has not been matched by a similar interst in the statics of plutonium reactions. Why there is so much more interest in the speed of the transportation than in the destination is not clear. It is possible, however, that 
the study of the statics of plutonium reactions may lend perspective to the study of plutonium reaction kinetics in aqueous solutions [47]. For example, it has been common practice to measure the concentrations of three or more plutonium oxidation states while studying the kinetics of plutonium disproportionation reactions. Where instrumental techniques allow accurate assay of three or more plutonium oxidation states in nonequilibrium circumstances, this is certainly a useful idea, but it is not the only approach, and perhaps not even the best approach [48].

During the course of a disproportionation reaction, the sum of the concentrations of all the plutonium oxidation states is always equal to the initial concentration of plutonium. Where the letters $W, X, Y$, and $Z$ symbolize trivalent, tetravalent, pentavalent, and hexavalent plutonium, respectively, this condition may be represented by Equation 1, in which $\mathrm{T}$ represents the total concentration of plutonium.

$$
W+X+Y+Z=T
$$

Equation 1 is a very good approximation to mass conservation in plutonium solutions; such accuracy is proably sufficient for most kinetic studies. Where $W, X, Y$, and $Z$ are interpreted as simple species - the usual case - it should be remembered that Equation I is only an approximation because it does not include such species as "activated complexes" which are always present in the plutonium solution. Another useful approximation is charge conservation as indicated in Equation 2:

$$
(3-N) W+(4-N) X+(5-N) Y+(6-N) Z=0
$$

where $\mathrm{N}$ represents the plutonium average oxidation number [47].

Beyond Equations 1 and 2, not a great deal may be said on an a priori basis about plutonium solutions not at equilibrium. However, Equations 1 and 2 represent two constraints upon plutonium kinetic systems. Given two more constraints, the four species of the nonequilibrium system may be completely determined. These additional constraints may be selected in various ways. Suppose, for example, that the popular disproportionation of pure tetravalent plutonium were reexamined in the customary, spectrophotometric manner. In this circumstance $N=4$, and Equation 2 becomes

$$
W=Y+2 Z
$$

Suppose further that the two extra constraints are arbitrarily selected as the instantaneous concentrations of trivalent plutonium and hexavalent plutonium, since the absorption spectra of these two species may be obtained without concentration changes during the course of measuring reference spectra. During the course of the disproportionation reaction, the instantaneous concentrations of trivalent and hexavalent plutonium are measured and, by use of Equation 3, they yield a value for the instantaneous concentration of pentavalent plutonium. Since $W, Y$, and $Z$ are then defined, the instantaneous concentration of tetravalent plutonium may be obtained by use of Equation 1. Hence, the entire system is characterized based on the instantaneous concentrations of two plutonium oxidation states. In principle, the instantaneous concentrations of trivalent and hexavalent plutonium may be obtained by light absorption measurements at two carefully selected wavelengths.

(Gary L. Silver) 


\section{Thorium-229}

A total of $11.2 \mathrm{mg}$ of thorium-229 was extracted and purified during this reporting period. Table 3 shows the results of mass spectrometric analyses of the feed material. In addition to the peaks indicated in

Table 3, peaks at masses 233, 234, and 237 were detected.

Table 3 - ISOTOPIC ANALYSES OF THORIUM BATCHES PROCESSED

\begin{tabular}{ccc} 
Batch* & Isotope & wt \\
\hline LP-4 & Th-232 & 93.2 \\
& Th-230 & 0.05 \\
& Th-229 & 6.8 \\
& Th-228 & 0.02 \\
L.P-5 & Th-232 & \\
& Th-230 & 96.3 \\
& Th-229 & 0.05 \\
& Th-228 & 3.7 \\
& & 0.01
\end{tabular}

* LP indicates low purity or material not previously processed at Mound Iaboratory.

Since both of these batches were removed from the same container, the differences in analytical results indicate a lack of homogeneity in the low-purity ORNL feed material. Also great difficulty was experienced during the extraction of these materials, the solvent extractant (di-secbutylphenylphosphonate) requiring several time-consuming cleanups with $\mathrm{Na}_{2} \mathrm{CO}_{3}$ each run. This indicates the presence of appreciable quantities of elements other than uranium and thorium in the feed material.

We evaluated the operation of the newly initiated, larger extraction equipment and concluded that the low purity of the feed material being used was the primary reason for the very slow extraction rates. The larger capacity of the re-designed extraction apparatus should compensate for the slower rate while also reducing the personnel gamma exposure. The new apparatus was designed, built, and put into operation during the reporting period.

A new 207-g batch of high-purity $\mathrm{UF}_{4}$ was dissolved and transferred into the extraction apparatus. This batch contains 11$14 \mathrm{mg}$ of thorium-229. It was anticipated from prior work that dissolution would require $1-3$ weeks but, using a premixed solution of $600 \mathrm{mI}$ of concentrated $\mathrm{HNO}_{3}$ and $90 \mathrm{~g}$ of $\mathrm{HBO}_{3}$, the dissolution actually occurred in less than 24 hours. The use of the pre-mix technique appears to significantly improve the processing of $\mathrm{UF}_{4}$ feeds. (Ronald L. Deaton)

\section{Thorium-230 and Protactinium-231}

Mound Laboratory recovers $\mathrm{Th}-230$ and $\mathrm{Pa}-231$ from a uranium mill by-product known as Cotter Concentrate and ships the products to Isotope Sales at ORNL. Previous reports contain detailed descriptions of the origin and character of the cotter Concentrate, as well as the facilities and development of the current recovery and purification processes [49-54]. Briefly, the process consists of leaching 20-25 liter batches of the solids with hot, concentrated $\mathrm{HNO}_{3}$ and then diluting and filtering off the insoluble residue. Uranium is removed from the 90-1iter batches of the filtrate by liquidliquid solvent extraction with 10\% DSBPP/ $\mathrm{CCl}_{4}$ (di-sec-butylphenylphosphonate in carbon tetrachloride) after which it is stripped from the organic with $0.005 \mathrm{M} \mathrm{HNO}_{3}$. Thorium is extracted from the filtrate by multiple contacts with $0.1 \mathrm{M}$ TOPO/ $\mathrm{CCl}_{4}$ (tri-n-octylphosphine oxide in carbon tetrachloride), each contact being followed by a $0.3 \mathrm{M} \mathrm{H}_{2} \mathrm{SO}_{4}$ strip. After the thorium 
has been removed from the filtrate, further contacts with TOPO/CCl 4 , each followed by a $0.5 \underline{M}$ oxalic acid strip, result in recovery of the protactinium. Uranium strip solutions are precipitated with ammonia and the precipitate collected for eventual return to the uranium processor. The thorium strip solutions are purified by oxalate precipitation and calcined to the oxide. Protactinium strip solutions are concentrated by evaporation, after which they are further purified by a series of precipitation and solvent extraction steps.

During this reporting period, 23 additional batches of cotter Concentrate were processed from Drums No. 169, 170 and 171. A shipment of $31.0 \mathrm{~g}$ of purified $\mathrm{Th}-230$ was made to Isotope Sales at ORNL in partial fulfillment of the FY-1977 quota. Thorium purity was equal to that of previous shipments. In addition, $31.26 \mathrm{~g}$ of purified Th-230 is on hand from recent production.

Unpurified protactinium-231 recovered from the 23 batches totals $108 \mathrm{mg}$. One additional evaporation batch has been extracted with TOPO and the protactinium stripped out with dilute HF. Strip solutions from previous extraction of five evaporation batches are being concentrated and purified. Depending on the level of impurities, phosphate precipitation from oxalic acid or recrystallization from sulfuric acid is being used. These methods have been described previously $[52,53]$. The latest analyses indicate that $135 \mathrm{mg}$ of $\mathrm{Pa}-231$ have been recovered from evaporation batches and this will be combined with $75 \mathrm{mg}$ of Pa-231 partially purified in previous operations.

The following additions were made to the analytical. instrumentation used for process control:
- A 4000-channel multichannel analyzer (MCA), to be used as a replacement for one of the 13-year-old MCA's now in use. The new MCA has been interfaced to the computer and is operational.

- An additional 8192 word add-on memory for the PDP 8/e minicomputer. (Now at the manufacturer for warranty repair.)

- A dual 8 "Floppy Disk" drive for the computer. (Now at the manufacturer for warranty repair.)

- A new Geli gamma detector which is now operational. Resolution is four times better, and detection efficiency is 10 times greater than for the unit previously used.

(Paul E. Figgins and Martin R. Hertz)

\section{Uranium-234}

Mound Laboratory has been separating and recovering uranium-234 of high isotopic purity from aged plutonium-238 materials for several years. The general chemical procedures have been described previously [ 55 5 $]$.

The initial separation of uranium from plutonium "cow" Bl4-0l by oxalate precipitation of the plutonium was completed this period. This was the third cycle of separation for B14-01, which contained $38.4 \mathrm{~g}$ of $\mathrm{Pu}-238 \mathrm{with}$ about one gram of high purity U-234. The BI4-0I "cow" was dissolved in hot, concentrated $\mathrm{HNO}_{3}$ containing 0.05-0.1M HF. The dissolved "cow" was divided into two equal parts which were adjusted to $2 \underline{\mathrm{M}} \mathrm{HNO}_{3}$ using $\mathrm{NH}_{4} \mathrm{OH}$. The plutonium in each half was precipitated as the oxalate with dimethyloxalate using $\mathrm{H}_{2} \mathrm{O}_{2}$ as reductant for plutonium. The 
oxalate precipitates were collected by vacuum filtration and combined with the oxalate precipitates from the initial separation of B14-02 which was described in the last report [56].

The combined precipitates were designated C14-0, calcined to the oxide at $400-500^{\circ} \mathrm{C}$, and returned to plutonium "cow" storage. The " $C$ " designation was used to indicate that a third separation cycle had been performed. The uranium-rich filtrates from both B14-02 and B14-01 precipitations were combined and evaporated to concentrate the volume and to destroy the oxalate. The concentrated filtrate was designated c14-filtrate, and is being held for intermediate processing.

The initial separation has been completed for combined plutonium "cows" A9-0 and A12-0. It was the second uranium separation cycle for these "cows", which contained $29.9 \mathrm{~g}$ of $\mathrm{Pu}-238 \mathrm{with}$ about $1.7 \mathrm{~g}$ of high purity U-234. To consolidate "cow" material, the oxalate precipitate was calcined to the oxide with oxalate precipitate Al3-0 (9.1 g of $\mathrm{Pu}-238)$ which was processed about two years ago. The oxide was designated A18-0, and returned to plutonium "cow" storage. The "A" designation was retained because A13-0 had been through just one separation cycle. The uranium-rich filtrate from precipitation of $A 9-0$ and $A 12-0$ was designated Al8-filtrate and is being held for intermediate processing.

The initial separation has been completed for combined plutonium "cow" $222 \mathrm{HO}$ and $727 \mathrm{HO}$. It was the second uranium separation cycle for this material, which contained $15.8 \mathrm{~g}$ of $\mathrm{Pu}-238 \mathrm{with}$ about $1.1 \mathrm{~g}$ of high purity U-234. The oxalate precipitate was calcined to the oxide, des- ignated B16-0, and returned to plutonium "cow" storage. The uranium-rich filtrate was designated Bl6-filtrate and is being held for intermediate processing.

The initial separation is in progress for plutonium "cow" $z_{1} 10-01$. It is the second uranium separation cycle for this "cow", which contains $40.2 \mathrm{~g}$ of $\mathrm{Pu}-238$ with about 1.69 of high purity U-234. This "cow" was divided into two parts for the precipitation. The oxalate precipitate is ready to be calcined, and the uraniumrich filtrate is ready to be evaporated.

The intermediate processing of the uraniumrich Al0-P filtrate has been completed. The initial separation of this batch was described in a previous report [57]. The A10-P filtrate was salted to $1.6 \mathrm{M}$ with $\mathrm{Al}\left(\mathrm{NO}_{3}\right)_{3}$ and adjusted to $0.3 \mathrm{M} \mathrm{HNO}_{3}$ with $\mathrm{NH}_{4} \mathrm{OH}$. This salted feed solution was loaded onto a column of Dowex $1 \times 4$ anion exchange resin (nitrate form) which had been preconditioned with $1.6 \underline{\mathrm{M}} \mathrm{Al}\left(\mathrm{NO}_{3}\right)_{3} /$ $0.3 \mathrm{M} \mathrm{HNO}_{3}$ reagent. After loading of the feed, the column was washed with $1.6 \mathrm{M} \mathrm{Al}\left(\mathrm{NO}_{3}\right)_{3} / 0.3 \mathrm{M} \mathrm{HNO}_{3}$ reagent and the uranium eluted with $7 \underline{\mathrm{M}} \mathrm{HNO}_{3}$. Finally, the plutonium was eluted with $0.35 \mathrm{M} \mathrm{HNO}_{3}$ containing hydroxylamine nitrate as a reductant for plutonium. Alpha pulse height analysis indicated a uranium fraction of $4.4 \mathrm{~g}$ of $\mathrm{U}-234$ with $850 \mathrm{ppm} \mathrm{Pu}-238$, which represents an improved decontamination factor over recent operations. Typically, 1500-25(0 ppm plutonium is expected at this stage.

Prior to the intermediate exchange run described above, a new resin column was installed. The diameter of the new column was $4 \mathrm{~cm}$ as compared to $3 \mathrm{~cm}$ for the old column. Additional resin was used to maintain the same bed height of approx- 
imately $17 \mathrm{~cm}$, which increased the volume. of resin by a factor of 1.8. It was anticipated that washing and eluting volumes would increase by the same factor and slow the operation if the same flow rate was used. In order to compensate for this, the flow rate was increased from 4 to $8 \mathrm{ml} /$ min. In fact, the washing and eluting volumes increased by a factor of 1.5. For this one run, the net effect of increased resin volume and increased flow rate was a slight savings in time and an improved decontamination factor. Further runs will be required to verify or disprove these observations. The increase in resin volume might also increase the uranium capacity per run, but this has not been tested. (Perle L. Keister) 


\section{Separation Research}

\section{Liquid Thermal Diffusion}

The compounds which were. studied in previous work on liquid phase thermal diffusion were normal liquids with critical temperatures well above the estimated hot wall temperature of the experimental column [58]. The reduced thermal diffusion factor, defined by

$$
\alpha_{0}=\alpha_{\Gamma}\left(\frac{m_{1}+m_{2}}{m_{1}-m_{2}}\right)
$$

where $\alpha_{T}$ is the thermal diffusion factor, and $m_{1}$ and $m_{2}$ are the molecular weights, is thought to be relatively constant. Thus, there is some advantage in selecting compounds of the lowest possible molecular weight to be used as working eluids for isotopic separation. In many cases such compounds will have critical temperatures near or below the hot wall temperature. Two such compounds were selected for experimental study. Methyl chloride $\left(\mathrm{T}_{\mathrm{c}}=\right.$ $416 \mathrm{~K})$ was chosen for chlorine isotope separation, and sulfur dioxide $\left(\mathrm{T}_{\mathrm{c}}=431 \mathrm{~K}\right)$ was chosen for ${ }^{34} \mathrm{~S}$ separation.

Equilibrium separation factors were measured for both materials in one of the $60-\mathrm{cm}$ prototype columns, for which the characteristics and operating conditions are given in Table 4. Experimental pressures were chosen to be well in excess of the critical pressures of the two compounds in order to preclude a phase boundary within the column. As Table 4 indicates, the estimated hot wall temperature for the methyl chloride experiment was slightly higher than $\mathrm{T}_{\mathrm{c}}$; thus, the fluid within the separation gap passed from a normal liquid at the cold wall to a compressed gas at the hot wall without a change of phase.

Experimental results are given in Table 5 . For methyl chloride the separation is given for the mass 50 /mass 52 pair representing $\mathrm{CH}_{3}{ }^{3} \mathrm{Cl}$ and $\mathrm{CH}_{3}{ }^{37} \mathrm{Cl}$. For sulfur dioxide, results are for the mass $64 /$ mass 66 pair representing ${ }^{32} \mathrm{SO}_{2}$ and ${ }^{34} \mathrm{SO}_{2}$ with a small contribution from ${ }^{32} \mathrm{~S}^{16} \mathrm{O}^{16} \mathrm{O}$ and ${ }^{32} \mathrm{~S}^{16} \mathrm{O}^{18} \mathrm{O}$. Thermal diffusion factors for the several experiments were calculated using the relationship

$$
\alpha_{r}=\left(K_{c}+K_{d}\right) \text { ln } q / \xi L
$$

where $K_{c}$ is the convective remixing coefficient, $K_{d}$ is the diffusive remixing coefficient, $q$ is the measured separation factor, and 1 is the length of the column. The quantity $\xi$, which can be calculated from theory, is defined such that

$$
\mathrm{H}=\bar{\alpha}_{T} \bar{\xi}
$$

where $H$ is the initial transport coefficient. (See reference 58 for details.)

Table 4 - DIMENSIONS AND OPERATINGCONDITIONS OF THE EXPERIMENTAL LIQUID THERMAL DIFFUSION COLUMN

Dimensions, $\mathrm{cm}$

$\begin{array}{lc}\text { Hot wall radius } & 1.2536 \\ \text { Cold wall radius } & 1.279 \\ \text { Length } & 60.96\end{array}$

Pressure, atm

$\mathrm{CH}_{3} \mathrm{Cl}$

137

$\mathrm{SO}_{2}$

$\mathrm{SO}_{2}$

Hot wall temperature, ${ }^{\circ} \mathrm{C}$ $\mathrm{CH}_{3} \mathrm{Cl}$

150.3

$\mathrm{SO}_{2}$

148.9

Cold wall temperature, ${ }^{\circ} \mathrm{C}$

$\mathrm{CH}_{3} \mathrm{Cl}$

70.4

$\mathrm{SO}_{2}$

75.4

Steam temperature, ${ }^{\circ} \mathrm{C}$

164.3

Cooling water temperature, ${ }^{\circ} \mathrm{C} \quad 15$ 
Table 5 - RESULTS OF ISOTOPIC SEPARATION

EXPERIMENTS WITH METHYL CHLORIDE AND SULFUR DIOXIDE

\begin{tabular}{llll} 
& \multicolumn{1}{c}{$\mathrm{CO}_{2}$} & \multicolumn{1}{c}{$\mathrm{SO}_{2}$} \\
$\ln \mathrm{g}$ & 0.456 & $\frac{(89.5 \mathrm{~atm})}{0.205}$ & $-\frac{(136 \mathrm{~atm})}{0.246}$ \\
$\xi_{,} \mathrm{g} / \mathrm{sec}$ & 0.00589 & 0.00979 & 0.00788 \\
$\mathrm{~K}_{\mathrm{c}}, \mathrm{g}-\mathrm{cm} / \mathrm{sec}$ & 0.0225 & 0.0637 & 0.0425 \\
$\mathrm{~K}_{\mathrm{d}}, \mathrm{g}-\mathrm{cm} / \mathrm{sec}$ & $3.23 \times 10^{-5}$ & $3.81 \times 10^{-5}$ & $3.68 \times 10^{-5}$ \\
$\alpha_{\mathrm{r}}$ & 0.0286 & 0.0219 & 0.0218 \\
$\alpha_{0}$ & 1.46 & 1.42 & 1.42
\end{tabular}

In order to evaluate the theoretical expressions for column transport coefficients it is necessary to have tabulated values for the density, viscosity, thermal conductivity, and self-diffusion coefficient covering the range of temperatures between the cold and hot walls of the column. At the estimated hot wall temperature both fluids approached the supercritical, dense fluid region of the phase diagram. It was no surprise to find that the available physical property data for these compounds are incomplete in this region. The property data available in the literature, chiefly for normal liquid conditions, were extended by several estimation techniques to cover the required range from 20 to $160^{\circ} \mathrm{C}$ The composite results are given in Tables 6 and 7 . Details concerning the literature data and the estimation techniques are given below.

Density - Good PVT data covering the compressed gas and liquid regions for both compounds were published by J. J. Mcketta and coworkers $[59,60]$; however, the minimum temperature at which liquid densities could be interpolated was $50^{\circ} \mathrm{C}$. Values at lower temperatures were obtained by extrapolation using published values of saturated liquid densities [61] for guidance. The estimated overall error in the density values given in Tables 6 and 7 is $0.5 \%$.

Viscosity - There are three sets of viscosity data for saturated liquid methyl chloride. Those of stakelbeck and Benning [62] are in substantial disagreement with the very old (1894) data of deHaas [61]. The compilers of the TPRC tables [6\%], who were apparently unaware of the deHaas data, extended the Benning and Stakelbeck data to an estimated value at the critical point by use of the equation

$$
\ln \eta=-2.546+348.6 / \mathrm{T}
$$

where $\eta$ is the viscosity in $\mathrm{CP}$, and $\mathrm{T}$ is the temperature in $\mathrm{K}$. At $50^{\circ} \mathrm{C}$ the TPRC curve differs from the deHaas curve by a factor of 1.7 .

One might be inclined to dismiss the old deHaas data; however, Figure 17 shows that the slope of the TPRC-Benning-. Stakelbeck curve differs significantly from the corresponding slopes for the other three chlorinated methanes. The deHaas data, in contrast, appear to form approximately the same slope as the other compounds. The generally reliable stielThodos reduced-state correlation [ 63 ], which also supports the deHaas data, yields 


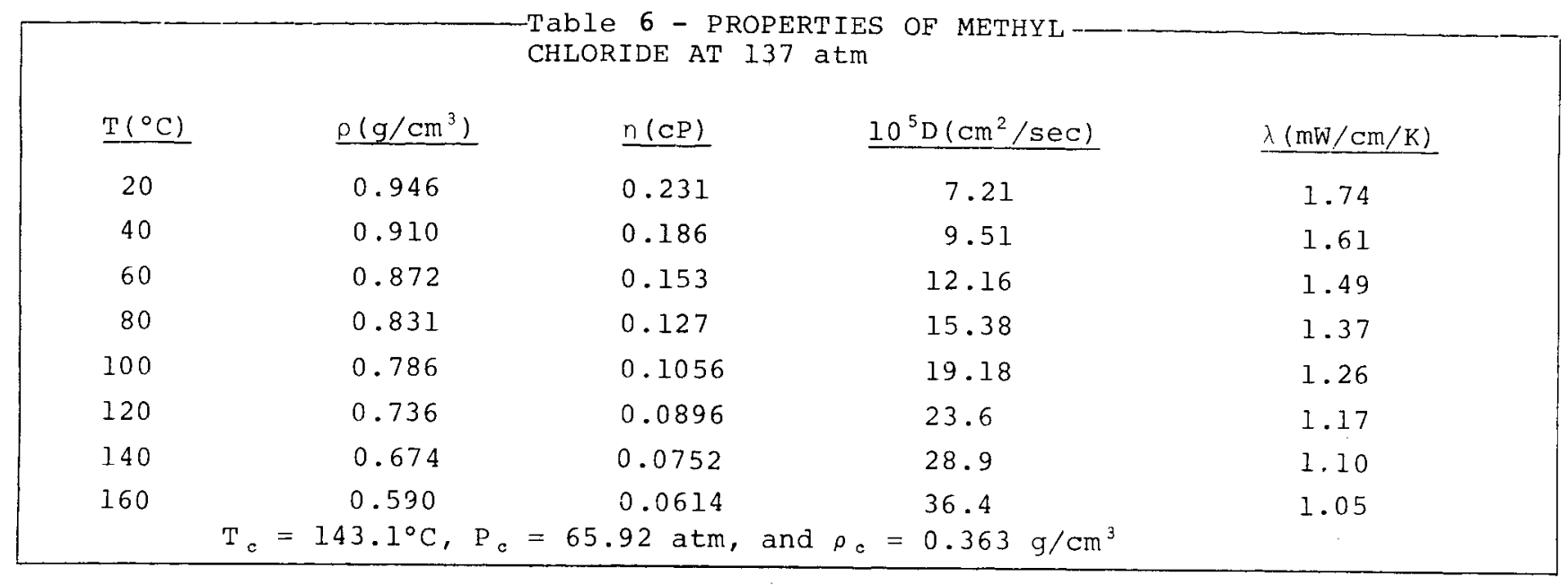

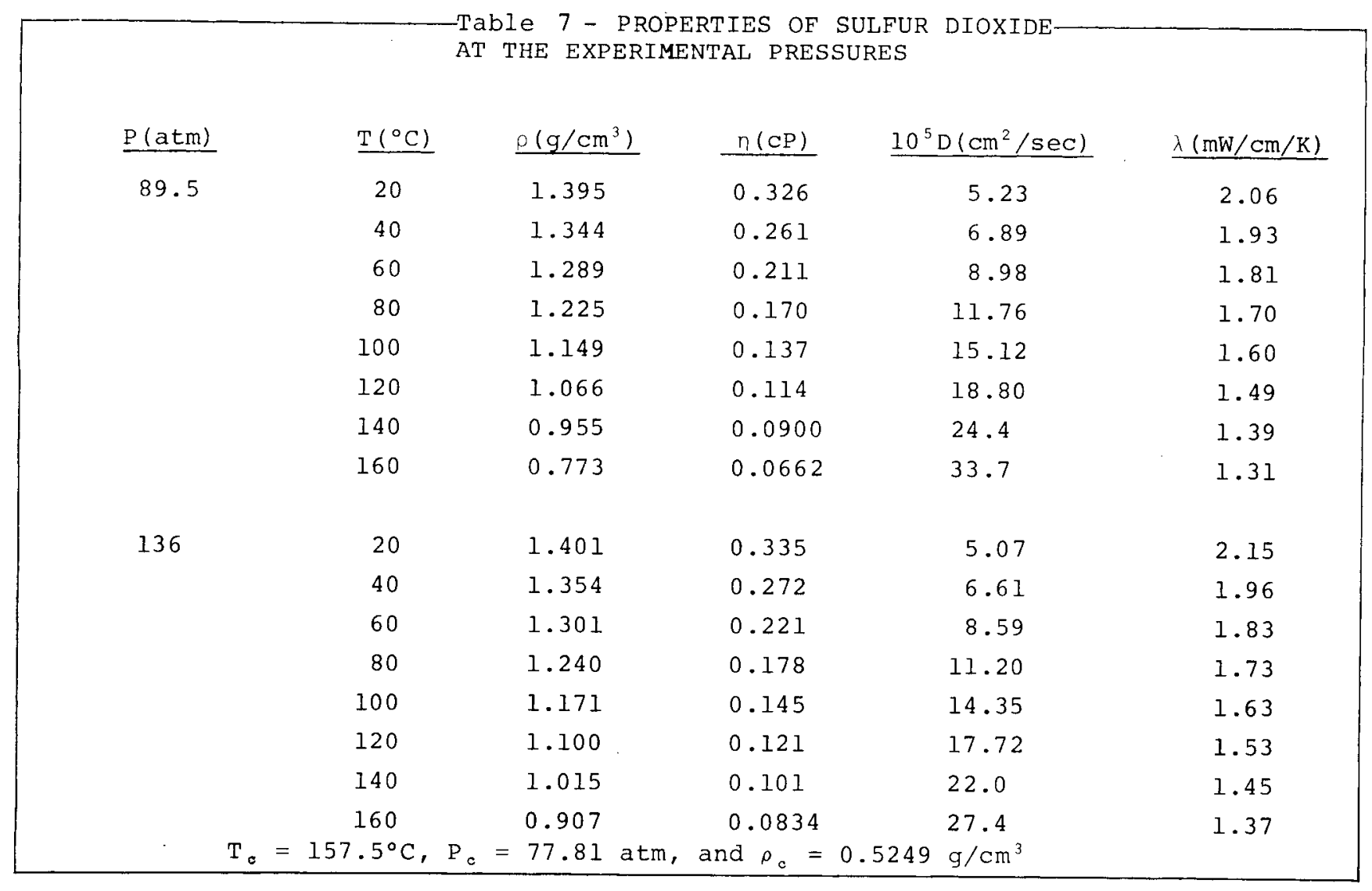




$$
\begin{aligned}
& \eta=n^{0}+\left(\frac{M^{1 / 2} P_{c}}{r_{c}{ }^{1 / 3}}\right)^{2 / 3}-\left\{\left[a+b \rho_{r}+c_{\rho_{r}}{ }^{2}+d_{\rho_{r}}{ }^{3}+e_{\rho_{r}}{ }^{4}\right]^{4}-10^{-4}\right\}(5) \\
& \text { where } n^{0}=\text { dilute gas viscosity, } c P \text {, at } \\
& \text { the same temperature } \\
& \mathrm{M}=\text { molecular weight } \\
& \mathrm{P}_{\mathrm{C}}=\text { critical pressure, atm } \\
& \mathrm{T}_{\mathrm{C}}=\text { critical temperature, } \mathrm{K} \\
& \rho_{r}=\text { reduced density }=\rho / \rho_{c} \text { where } \\
& \rho \text { is the density } \\
& \mathrm{a}=0.10230 \\
& \mathrm{~b}=0.023364 \\
& \mathrm{c}=0.058533 \\
& \mathrm{~d}=-0.040758 \\
& e=0.0093324
\end{aligned}
$$

Equation 5 is valid for compressed gases and liquids in the reduced density range from 0.1 to 3.0. Reid and sherwood [63] state that errors are usually less than 10 to 15 응 if experimental densities are used.
As Figure 17 indicates, the stiel-Thodos equation is in reasonably good agreement with the deHaas data and with the lowest temperature data of the Stakelbeck-Benning group. Accordingly, the stiel-Thodos equation was chosen to calculate methyl chloride viscosities at the experimental pressure of $137 \mathrm{~atm}$. If the TPRC extrapolation is ignored, then the values listed in Table 6 are probably correct within $\pm 15 \%$

The very limited data for saturated liquid sulfur dioxide $[61,64]$, which are plotted in Figure 18, are consistent with the Stiel-Thodos equation; therefore, the Stiel-Thodos equation was also used to predict sulfur dioxide viscosities at the experimental pressures of 136 and $89.5 \mathrm{~atm}$. Figure 18 shows the calculated curves for the saturated liquid and the two curves for the compressed liquid. The error is estimated to be $\pm 15 \%$ or less.

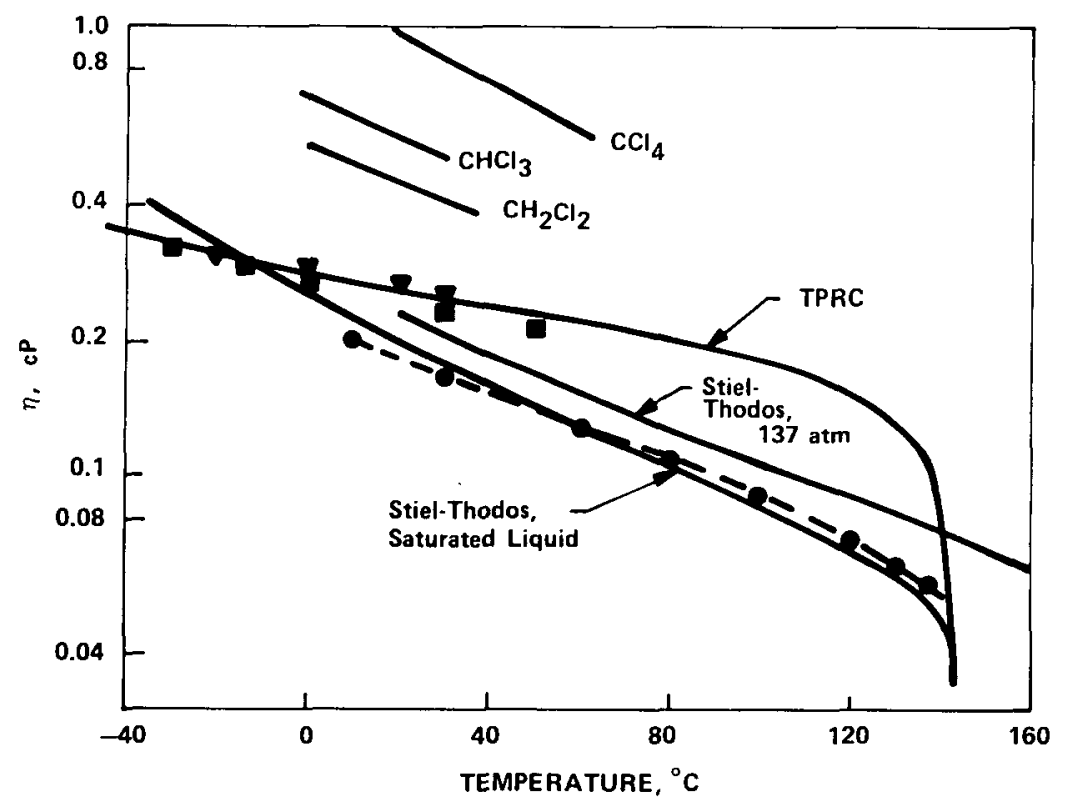

FIGURE 17 - Viscosity of methyl chloride. The e's are the data of deHads; the $\mathbf{a}^{\prime} \mathbf{s}$, the data of Benning; and the $\boldsymbol{\Delta}^{\prime} \mathrm{s}$, the data of Stakelbeck. 

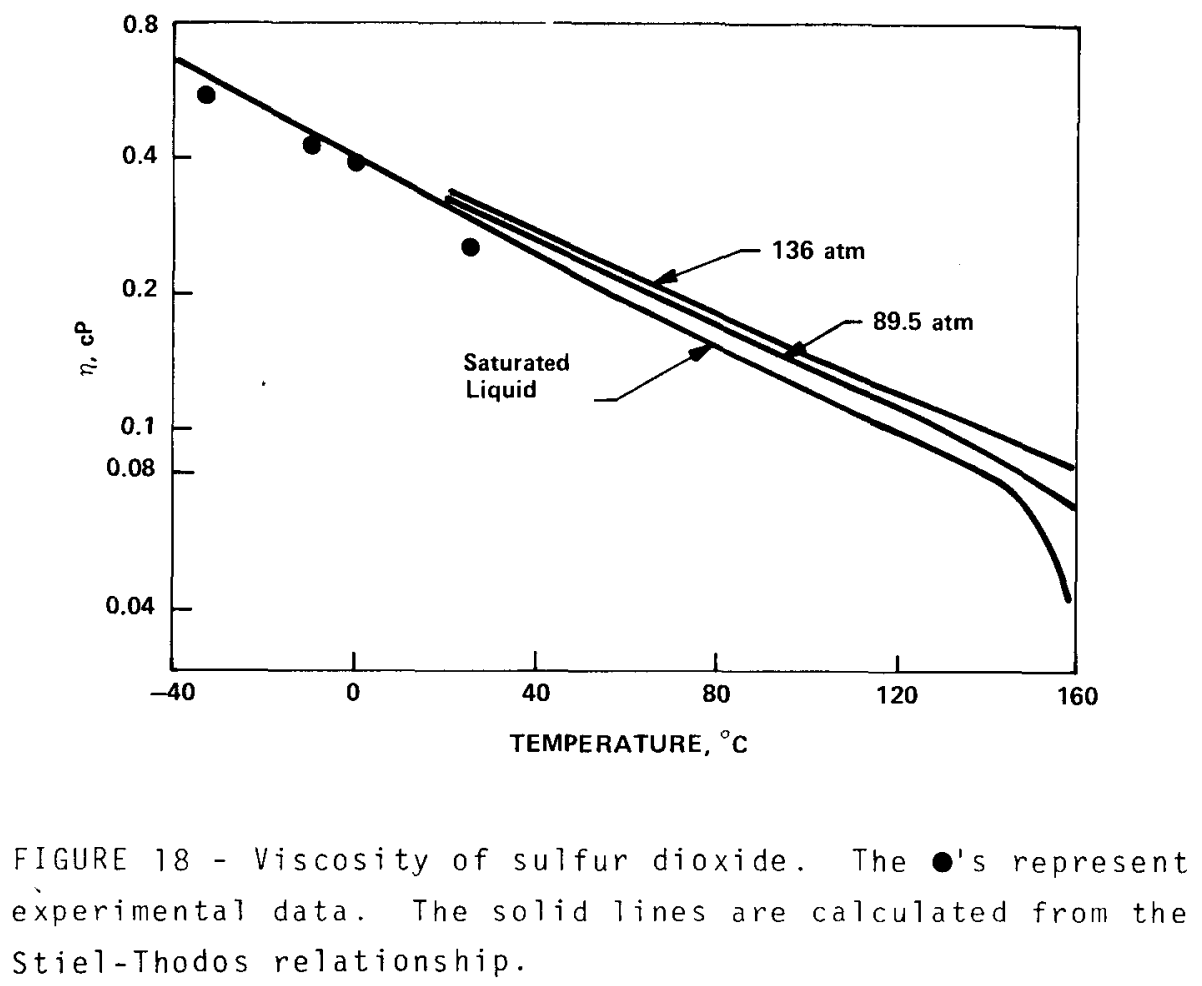

Self-Diffusion Coefficient - Self-diffusion data do not appear to exist for either compound. In the normal liquid region the diffusion coefficient can be predicted from the viscosity according to a method described by Ertl and Dullien [6 5]. If one defines

$$
\phi=\frac{n V D}{R T}
$$

then $\phi$ passes through a minimum as a function of temperature. The minimum is given by

$$
\phi_{\min }=a+b V_{c}^{2 / 3}
$$

where $V_{c}$ is the critical molar volume. At temperatures other than the temperature of the minimum, $\phi$ is given by

$$
\phi=\dot{\phi}_{\min }\left[\frac{\mathrm{A}}{\mathrm{T}^{4}}+\mathrm{B}+\mathrm{CT}_{r}\right]^{2}
$$

According to Ertl and Dullien the following are the parameters in the preceding equations :

$$
\begin{aligned}
& \mathrm{a}=-1.42 \\
& \mathrm{~b}=0.152 \\
& \mathrm{~A}=2.55 \times 10^{-3} \\
& \mathrm{~B}=0.179 \\
& \mathrm{C}=0.35
\end{aligned}
$$

A careful examination of their Figure 10 [65], which is a plot of Equation 7, suggests that the above value of a is incorrect. A better value seems to be

$$
a=-1.04
$$

One would not expect the above to be useful in the critical region. Accordingly, a study was made of published data on the self-diffusion and viscosity of methane in the critical region in order to develop 
some preliminary guidelines for estimation $[66,67]$. At least for methane, $\phi$ was discovered to be dependent only on the reduced density over a temperature range of from $154.5 \mathrm{~K}$ to $256.7 \mathrm{~K}$. This relationship, which is shown in Figure 19, smoothly approaches the minimum value calculated according to Equation 7 with $a=-1.04$. Also shown in Figure 19 is a plot of the smoothed ratio defined by

$$
r=\phi\left(\rho_{r}\right) / \phi_{\min }
$$

The values of the diffusion coefficients for sulfur dioxide and methyl chloride which appear in Tables 6 and 7 were calculated from the similarly tabulated viscosities according to

$$
D=\frac{R T}{\eta V} r\left(p_{r}\right) \phi_{\min }
$$

Probable errors in the estimated diffusion coefficients cannot be predicted; however, they are clearly larger than the errors in the viscosity. More work will be done on

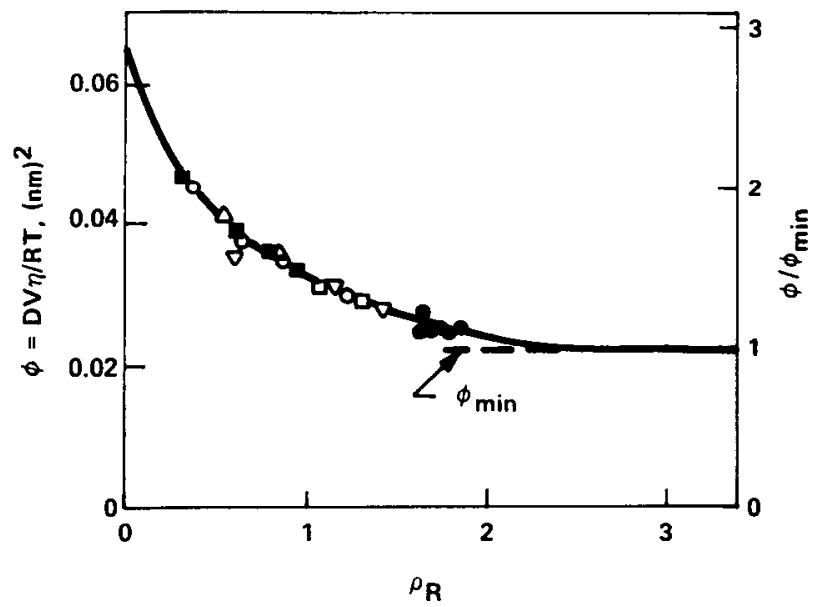

FIGURE 19 - Self-diffusion of methane in the liquid and critical regions. estimating the diffusion coefficient. A complete search of the literature has not been performed. It is known, for instance, that data exist for other substances, such as $\mathrm{H}_{2}$ and $\mathrm{CO}_{2}$. It would be highly desirable to support Figure 19 with additional data.

Thermal Conductivity - The TPRC tables [6 2] contain smoothed extrapolated values of the thermal conductivity of saturated liquid methyl chloride and sulfur dioxide. But, there does not appear to be any technique available for predicting the conductivity except at saturation or at near critical conditions. In order to estimate the required values, a corresponding-state technique was developed based on the Pitzer acentric factor [63], $w$, and the thermal conductivity at a reference point, arbitrarily chosen as $\mathrm{T}_{\mathrm{R}}=0.7$ and $\mathrm{P}_{\mathrm{R}}=1$. Thus

$$
\lambda=\lambda_{O} B\left(\mathrm{~T}_{R}, \mathrm{P}_{\mathrm{A}}, \omega\right)
$$

where $\lambda$ is the thermal conductivity and $\lambda_{0}$ is the thermal conductivity at the reference point. Data from the Jamieson, Irving, and Tudhope compilation [68] for methane, ethanol and $n$-butane were interpolated to get plots similar to Figure 20 for each of the three experimental reduced pressures. Values of $\beta$ were taken from a curve interpolated to the value of $\omega$ for methyl chloride or sulfur dioxide. The TPRC tables and fourth plot for $\mathrm{P}_{r}=0$ were used to estimate values of $\lambda_{0}$. Values of $\omega$ were taken from Reference 63 .

The resulting thermal conductivities, for which the probable error cannot be estimated, are given in Tables 6 and 7 . Fortunately, the calculated thermal diffusion column coefficients are rather insensitive to the thermal conductivity; thus, relatively large errors can be tolerated.

(W. M. Rutherford) 


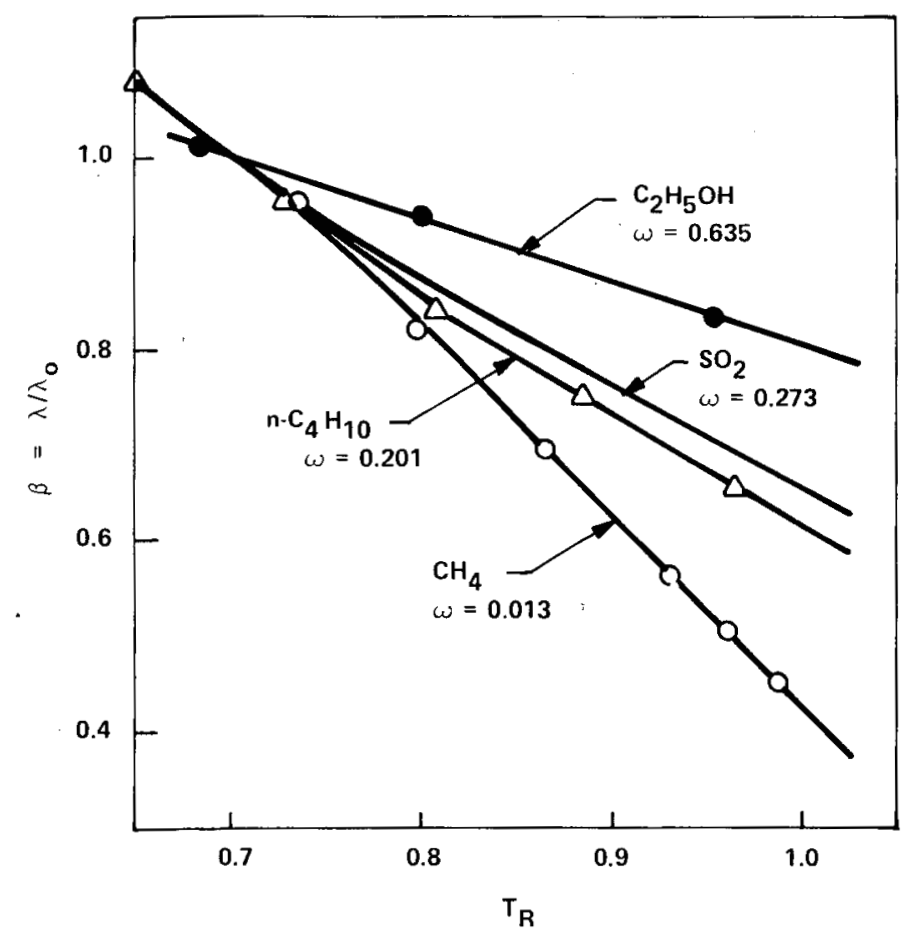

FIGURE 20 -Correlation of the thermal conductivity ratio at a reduced pressure of 7.15 .

\section{Molecular Beam Scattering}

The new low-temperature nozzle beam source was installed in the secondary arm of the molecular beam chamber. The source was adapted for connection of a Cryotip refrigerator similar to that on the primary beam source. When the new Cryotip was found to have a severe internal leak between the $\mathrm{H}_{2}$ and $\mathrm{Ne}$ cooling loops and the chamber vacuum, it was removed from the chamber and sent to Air Products Inc. for repairs.

Final laser alignment of the primary and secondary chamber arms with the detector platform was completed. The quadrupole detector was installed on the rotary platform and adjusted for optimum alignment and resolution.

Ideal skimmers from centre d' Etudes Nucleaires de Saclay, France and new precision nozzles from a local vendor were received and installed on both sources for an expected increase in beam intensity of up to one order of magnitude. The expected increase is largely due to a uniquely sharp leading edge and optimum included cone angle on the new skimmers.

The new, low-temperature target cell, described previously [69], was assembled and installed in the beam chamber. The system is currently in the pumpdown mode prior to achieving satisfactory stable operating conditions at less than $1 \times 10^{-7}$ torr. When this state is reached, an experinent using two crossed beams will be performed to determine the capability of the detection system for measuring differential scattering. The chamber will then be adjusted for measuring the total scattering/ cross sections for a new scattering system, prior to the reinstallation of the repaired Cryotip refrigerator on the secondary beam source. (Robert W. York) 


\section{Transport Properties}

THERMAL DIFFUSION FACTORS FOR ${ }^{3} \mathrm{He}-{ }^{4} \mathrm{He}$

Three experiments were conducted in the two-bulb apparatus at $4.2 \mathrm{~K}$ as part of the project to determine the thermal diffusion factor, $\alpha_{\mathrm{I}}$, for ${ }^{3} \mathrm{He}-{ }^{4} \mathrm{He}$ at very low temperatures. In addition, a calibration was made between a) the platinum resistance thermometer embedded in the block ( $\mathrm{T}_{\mathrm{AC}}$ ) versus the platinum resistor standing free in the gas $\left(\mathrm{T}_{\mathrm{OBS}}\right), 15<\mathrm{T}_{\mathrm{AC}}<100 \mathrm{~K}$, and b) the calibrated carbon resistor embedded in the block versus the above free-standing platinum resistor, $4.2<\mathrm{T}_{A C T}<15 \mathrm{~K}$.

As mentioned in a previous report [70], self-heating of the platinum resistor gave an observed temperature reading slightly higher than the actual temperature of the problem resulted from the insensitivity of the platinum resistor below $15 \mathrm{~K}$. Here the lead wire and contact resistance become significant compared to the thermometer resistance. For this reason the calibrated carbon resistor was used to measure the actual temperature for several arbitrary bridge settings for the platinum resistor $\left(\mathrm{R}_{\mathrm{pt}}<0.5 \Omega\right)$. The actual temperatures were higher than the bridge settings indicated they should be, resulting in a significant, but not accurately determinable, shift of the abscissa value to the left.

Figure 22 shows the results of these translations for the low-temperature portion of of the $\ln q$ vs. In $\left(T^{*} / T_{c}\right)$ curve. The arrows denote the postulated shifts in Weissman's data. The three clustered circles at $4.2 \mathrm{~K}$ should probably not be shifted since, for these experiments, the

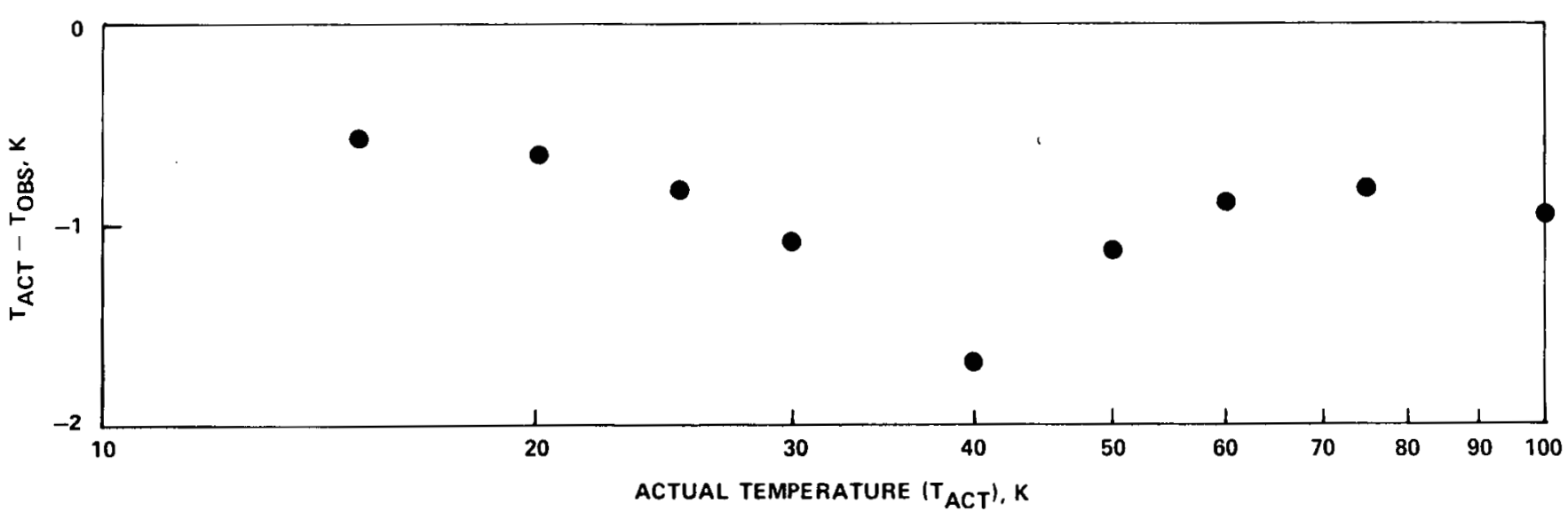

FIGURE 21 - Temperature correction for self-heating of the platinum resistance thermometer. free-standing in the cold bulb

can. The calibration curve is shown in Figure 21. These corrections were applied to the observed readings given by Weissman, and corrected values of the abscissa, In $\left(T_{H / T_{c}}\right)$, were examined. The corrections caused a slight shift of points to the right but not enough to seriously affect the results for $\mathrm{T}_{\mathrm{c}}>15 \mathrm{~K}$. A more serious cold bulb was immersed in liquid helium with an exchange gas in the vacuum jacket making it very improbable that a temperature difference of more than a fraction of a degree could be sustained. Neither the thermometer nor the heater was needed, the cold temperature being taken as that of the liquid helium bath. 
The results of the three recent experiments are shown by the three squares in Figure 22 .

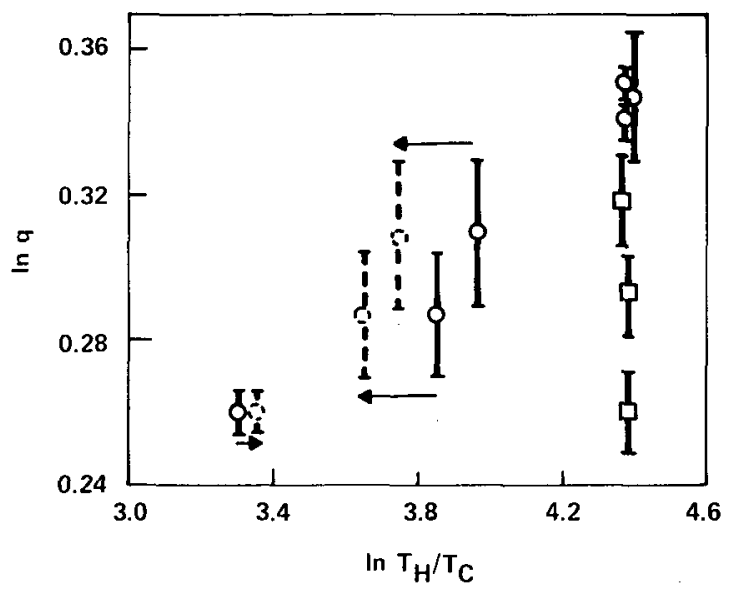

FIGURE 22 - Separation factor for equimolar ${ }^{3} \mathrm{He}-{ }^{4} \mathrm{He}$ as measured in the two-bulb apparatus at very low temperatures. The o's represent previous data by Weissman corrected for thermometry errors; the a's, present results.

There is obviously a problem with the reproducibility in the present work. The best hypothesis at this point in time is that the major difficulty lies in the relaxation time of the experiment which is given in the expression

$$
T=\frac{I_{A}}{\bar{A}}\left(\frac{T_{c}}{V_{c}}+\frac{T_{H}}{V_{H}}\right)^{-I}<\frac{\bar{T}}{\bar{D}}>
$$

Here $L$ is the length of the connecting tube, $A$ is its area, and $T_{H}, V_{H}, T_{C}$ and $V_{C}$ are the temperatures and volumes of the hot and cold bulbs, respectively. The quantity $\langle\overline{\mathrm{T}} / D\rangle$ is the average temperature in the connecting tube with the diffusion coefficient for ${ }^{3} \mathrm{He}-{ }^{4} \mathrm{He}$ corresponding to the average temperature. Weissman assumed $\overline{\mathrm{T}}=1 / 2\left(\mathrm{~T}_{\mathrm{C}}+\mathrm{T}_{H}\right)$, determined $7 \mathrm{~T}$, and then (in most cases) added a few hours to obtain the duration of the experiment. Another school of thought holds that the log mean temperature provides a more accurate rep- resentation of the average temperature of a gas in a temperature gradient; e.g., $\overline{\mathrm{T}}=\mathrm{T}_{H} \mathrm{~T}_{C} /\left(\mathrm{T}_{\mathrm{H}}-\mathrm{T}_{\mathrm{C}}\right)$ ln $\mathrm{T}_{H} / \mathrm{T}_{C}$. Use of this expression yields considerably lower average temperatures and consequently, much smaller diffusion coefficients.

The present results are interpreted in terms of the relaxation times for the experiments, and it is postulated that the log mean temperature more accurately represents the true condition of the gas in the apparatus. Table 8 illustrates this argument based on the duration of the experiments expressed as multiples of the calculated relaxation times for the three present experiments.

\begin{tabular}{|c|c|c|}
\hline \multicolumn{3}{|c|}{$\begin{array}{l}\text { Table } 8 \text { - DURATION OF EXPERIMENT- } \\
\text { Here } \tau \text { represents the relaxation } \\
\text { time calculated from tire arithme- } \\
\text { tic average, and } \tau \text { ' represents } \\
\text { that calculated from the log mean } \\
\text { average temperature. }\end{array}$} \\
\hline Lowest Point & $7 \tau$ & $2 \tau^{\prime}$ \\
\hline Middle Point & $12 \tau$ & $3 \tau^{\prime}$ \\
\hline Highest Point & $25 \mathrm{~T}$ & $7 \tau^{\prime}$ \\
\hline
\end{tabular}

Because of the exponential approach to equilibrium, then, approximately $7 \tau$ is the time necessary to approach the steady state within $1 \%$. This line of reasoning explains the trend in the present data. It does not, however, explain why Weissman's three data points at $4.2 \mathrm{~K}$ were so much higher; one of the recent experiments (middle $\square$ ) duplicated his $4.2 \mathrm{~K}$ experiments in all experimental parameters including the operating time. Future experiments will attempt to resolve this discrepancy.

(William L. Taylor) 


\section{References}

1. Mound Laboratory Activities for the Division of Physical Research:

January-June 1976, MLM-2354 (September $30,1976), \mathrm{p} .8$.

2. W. E. Keller, Helium-3 and Helium-4, Plenum Press, New York (1969), pp. 8590 .

3. L. Holborn and J. Otto, 2. Phys., 10, 367 (1922).

4. A. Michels and H. Wouters, Physica, 8, 923 (1941).

5. J. O. Herschfelder, C. F. Curtiss, and R. B. Bird, Molecular Theory of Gases and Liquids, J. Wiley and Sons, New York (1954), pp. 413-424.

6. D. E. Beck, Mol. Phys., 14, 311 (1968).

7. L.W. Bruch and I. J. McGee, J. Chem. Phys., 52, 5884 (1970).

8. J. M. H. Levelt-Sengers, M. Klein, and J. S. Gallagher, AEDC-TR-71-39 (1971).

9. J. M. Farrar and Y. T. Lee, J. Chem. Phys., 56, 5801 (1972).

10. A. L. J. Burginans, J. M. Farrar, and Y. T. Lee, J. Chem. Phys., 64, 1345 (1976).

11. B. E. Gammon, J. Chem. Phys., 64, 2556 (1976).

12. K. T. l'ang and J. P. Toennies, J. Chem. Phys., 66, 1496 (1977).

13. R. Ahlrich, R. Penco, and G. Scoles, Chem. Phys., 19, 119 (1977).
14. R. Aziz, private communication (to be published).

15. Mound Laboratory Activities for the Division of Physical Research: January-June 1976, MLM-2354 (Sept. 30, 1976), pp. 22-25.

16. Mound Laboratory Activities in Chemical and Physical Research: July-December 1976, MLM-2414 (May 4, 1977), pp. 11-12.

17. J. J. Reilly and R. H. Wiswall, Jr., Inorg. Chem., 13, 218 (1974).

18. J. Volkl and G. Alefeld in Diffusion in Solids-Recent Developments,

A. S. Nowick and J. J. Burton (ed.), Academic Press, New York, 1975, p. 232.

19. Mound Laboratory Activities for the Division of Physical Research: JanJune, 1975, MLM-2241 (Nov. 11, 1975), pp. $20-24$.

20. Mound Laboratory Activities for the Divison of Physical Research: JulyDecember 1975, MLM-2296 (May 16, 1976), pp. 20-22.

2]. R. C. Bowman, Jr., A. Attalla, and W. E. Tadlock, Int. J. of Hydrogen Energy, 1, 421 (1977).

22. H. Asano and M. Hirabayashi, Phys. Status Solidi A, 16, 69 (1973).

23. R. R. Arons, H. G. Bohn, and H. Lütgemeier, J. Phys. Chem. Solids, 35, 207 (1974).

24. H. Asano, Y. Abe, and M. Hirabayashi, Acta Metall., 24, 95 (1976). 
25. H. Asano, Y. Abe, and M. Hirabayashi, J. Phys. Scc. Japan, 41, 974 (1976).

26. R. C. Bowman, Jr., and A. Attalla, in Magnetic Resonance and RelaxationProceedings of the XIXth Congress Ampere, Heidelberg, September 1976, H. Brunner, K. H. Hausser, and D. Schweitzer (ed.), Groupement Ampere, Geneva, 1976, pp. 315-8.

27. R. M. Cotts, Ber. Bunsenges. Phys. Chem., 76, 760 (1972).

28. D. Rohy and R. M. Cotts, Phys. Rev. B, $1,2070(1970)$.

29. C. Korn and D. Zamir, J. Phys. Chem. Solids, 34, 725 (1973).

30. P. P. Davis et al., J. Less-Common Met., 49, 159 (1976).

31. C. Korn and D. Zamir, J. Phys. Chem. Solids, 31,489 (1970).

32. A. Schmolz and F. Noack, Ber. Bunsenges. Phys. Chem., 78, 339 (1974).

33. B. Pedersen, T. Krogdahl, and

O. E. Stokkeland, J. Chem. Phys., 42, 72 (1965).

34. H. Lütgemeier, R. R. Arons, and H. G. Bohn, J. Magn. Reson., 8, 74 (1972).

35. G. K. Schoep, N. J. Poulis, and R. R. Arons, Physica, 75, 297 (1974).

36. A. J. Maeland, J. Phys. Chem., 68, 2197 (1964).

37. D. G. Westlake et. al., Metall. Trans:, 1, 1861 (1970).
38. Y. Fukai and S. Kazama, Scr. Metall... 9, 1073 (1975).

39. Mound Laboratory Activities in Chemical and Physical Research: JulyDecember 1976, MLM-2414 (May 4, 1977), pp. 16-17.

40. G. C. Abell and R. C. Bowman, Jr., Phys. Lett., 60A, 353 (1977).

41. E. A. Knyazev, Zh. Fiz. Khim., 49:6, 1534 (1975).

42. G. L. Silver, J. Inorg. Nucl. Chem., 35, 1369 (1973).

43. G. L. Silver, Plutonium in Natural Waters, MLM-1870 (Dec. 16, 1971), $15 \mathrm{pp}$.

44. G. L. Silver, Radiochem. Radioanal. Letters, 9 : $\underline{5}, 315$ (1972).

45. Mound Laboratory Isotopic Power Fuels Programs: July-September 1973. MLM-2099 (Nov. 21, 1973), pp. 21-23.

46. T. M. Florence and G. E. Batley, Talanta, 24, 151 (1977).

47. Mound Laboratory Activities for the Division of Physical Research: JanJune 1976, MLM-2354 (Sept. 30, 1976), p. 26 .

48. C. Poitrenaud and A. G. Wain, Disproportionation of Plutonium IV in Concentrated Solutions of Plutonium in Perchloric Acid, AERE - R - 8359 (September 1976).

49. Mound Laboratory Activities for the Division of Physical Research: July- 
December 1973, MLM-2118 (February 28, 1974), pp. 32-35.

50. Mound Laboratory Activities for the Division of Physical Research: Jan.June 1974, MLM-2168 (August 30, 1974). pp. $31-35$.

51. Mound Laboratory Activities for the Division of Physical Research: Jan.June 1975, MLM-2241 (Nov. 11, 1975), pp. 29-3l.

52. Mound Laboratory Activities for the Division of Physical Research: JulyDecember 1975, MLM-2296 (May 14, 1976), pp. 25-29.

53. Mound Laboratory Activities for the Divison of Physical Resarch: Jan.June 1976, MLM-2354 (Sept. 30, 1976), pp. 32-35.

54. Mound Laboratory Activities in Chemical and Physical Research: JulyDecember 1976, MLM-2414 (May 4, 1977), pp. 19-20.

55. Mound Laboratory Activities for the Division of Physical Research: JulyDecember 1972, MLM-2013 (February 14, 1973), pp. 56-59.

56. Mound Laboratory Activities in Chemical and Physical Research: JulyDecember 1976, MLM-2414 (May 4, 1977), P. 21 .

57. Mound Laboratory Activities for the Division of Physical Research: Jan.June 1976, MLM-2354 (September 30 , 1976), p. 35 .

58. W. M. Rutherford, J. Chem. Phys., 59, 6061 (1973).
59. C. C. Hsu and J. J. MCKetta, J. Chem. Eng. Data, 9 , 45 (1964).

60. T. L. Kang et al., J. Chem. Eng. Data, 6,220 (1961).

61. Landolt-Börnstein PhysikalischChemische Tabellen, 5th Ed., Springer verlag, Berlin, 1936.

62. Y. S. Touloukian, S. C. Saxena, and P. Hestermans, Thermophysical properties of Matter, Vol. 3 and 11 , IFI/Plenum, New York, 1975.

63. R. C. Reid and T. K. Sherwood, The Properties of Gases and Liquids, 2nd Ed., McGraw-Hill Book Co., Inc., New York, 1966.

64. International Critical Tables, McGrawHill Book Co., Inc., New York, 1926.

65. W. Ertl and F. A. L. Dullien, AIChE J., 19, 1215 (1973).

66. R. Dawson, F. Khoury, and R. Kobayashi, AIChE J., 16, 725 (1970).

67. E. T. S. Huang, G. W. Swift, and F. Kurata, AIChE J., 12, 932 (1966).

68. D. T. Jamieson, J. B. Irving, and J. S. Tudhope, Liquid Thermal Conductivity, Her Majesty's Stationery office, Edinburgh, 1975.

69. Mound Laboratory Activities in Chemical and Physical Research: JulyDecember 1976, MLM-2414 (May 4, 1977), p. 29 .

70. Mound Laboratory Activities in Chemical and Physical Research: JulyDecember 1976, MLM-2414 (May 4, 1977), pp. 29-34. 


\section{Distribution}

\section{EXTERNAL}

Technical Report Library, Monsanto, St. Louis TID-4500, UC-4 and 22 (231)
J. A. Chacon, DAO
R. B. Craner, SLA
R. K. Flitcraft, MRC
W. J. Haubach, DBES
N. Haberman, DNRA
W. E. Moddeman
F. D. Stevenson, DBES
C. P. Sutter/R. N, Diebel, Atlantic Richfield
D. White
R. N. Zare

INTERNAL
V. L. Avona
R. C. Bowman, Jr.
W. T. Cave
R. J. Desando
R. E. Eppley
C. W. Huntington
B. E. Jepson
$\mathrm{L}$. $\mathrm{V}$. Jones
K. C. Jordan
C. M. Love
J. R. MCClain
E. D. Michaels
W. J. RoOS
W. M. Rutherford
R. A. Schwind
G. L. Silver
W. L. Taylor
H. L. Turner
R. E, Vallee
R. M. Watrous
Document Control
Library (15)
Publications (25) 